\title{
INHERENT AND EPISTEMIC UNCERTAINTY ANALYSIS FOR COMPUTATIONAL FLUID DYNAMICS SIMULATIONS OF SYNTHETIC JET ACTUATORS
}

\author{
Daoru Han ${ }^{1, *}$ E Serhat Hosder ${ }^{2}$ \\ ${ }^{1}$ Laboratory for Astronautical Plasma Dynamics, Department of Astronautical Engineering, 233 \\ Robert Glenn Rapp Engineering Research Building, University of Southern California, Los An- \\ geles, California 90089-1192, USA \\ ${ }^{2}$ Aerospace Simulations Laboratory, Department of Mechanical and Aerospace Engineering, \\ 290B Toomey Hall, Missouri University of Science and Technology, Rolla, Missouri 65409- \\ 0500, USA
}

Original Manuscript Submitted: 01/02/2014; Final Draft Received: 07/17/2014

\begin{abstract}
A mixed uncertainty quantification method was applied to computational fluid dynamics (CFD) modeling of a synthetic jet actuator. A test case, flow over a hump model with synthetic jet actuators, was selected from the CFDVAL2004 workshop to apply the second-order probability framework implemented with a stochastic response surface obtained from quadrature-based nonintrusive polynomial chaos. Three uncertainty sources were considered: (1) epistemic uncertainty in turbulence model, (2) inherent uncertainty in free stream velocity, and (3) inherent uncertainty in actuation frequency. Uncertainties in both long-time averaged and phase averaged quantities were quantified using a fourth-order polynomial chaos expansion. A global sensitivity analysis with Sobol indices was utilized to rank the importance of each uncertainty source to the overall output uncertainty. The results indicated that for the long-time averaged separation bubble size, the uncertainty in turbulence model had a dominant contribution, which was also observed in the long-time averaged skin-friction coefficients at three selected locations. The mixed uncertainty results for phase-averaged $x$-velocity distributions at three selected locations showed that the $95 \%$ confidence interval could generally envelop the experimental data. The Sobol indices showed that near the wall, the uncertainty in turbulence model had a main influence on the $x$-velocity. While approaching the main stream, the uncertainty in free stream velocity became a larger contributor. The mixed uncertainty quantification approach demonstrated in this study can also be applied to other CFD problems with inherent and epistemic uncertainties.
\end{abstract}

KEY WORDS: uncertainty quantification, polynomial chaos, stochastic response surface, computational fluid dynamics, synthetic jet actuators

\section{INTRODUCTION}

Synthetic jet actuators are one of the most frequently studied flow control configurations since they are highly promising in terms of realizing actual flow control system on an aircraft. In a typical synthetic jet actuator configuration, the jet is produced by a moving membrane that is built into the wall of the cavity. This jet is ejected out through an orifice that can be directly mounted on the control surface. The simplicity of the design obviates the need for complex ducting and packaging and hence a more attractive solution. Unique to synthetic jet, is also the fact that they are formed by the working fluid in the flow system in which they are employed. This results in an addition of momentum to the system without adding any mass, hence named "zero net-mass-flux jets." During the ejection half of the membrane motion, for a two-dimensional orifice, the flow separates at the sharp edges of the orifice and rolls into a pair

\footnotetext{
${ }^{*}$ Correspond to Daoru Han, E-mail: daoruhan@usc.edu, URL: http://www-scf.usc.edu/ daoruhan/
} 


\section{NOMENCLATURE}

$c \quad$ characteristic reference length

$C_{f} \quad$ skin friction coefficient

$C_{p} \quad$ Pressure coefficient

$\mathrm{CoV}$ coefficient of Variation

$D \quad$ statistical variance

$f \quad$ frequency $(\mathrm{Hz})$

$K \quad$ factor in s-a turbulence model

$n \quad$ number of random variables

$N_{t} \quad$ number of output modes

$p \quad$ pressure $\left(\mathrm{N} / \mathrm{m}^{2}\right)$ or order of PCE

$S \quad$ Sobol index

$S_{T} \quad$ total Sobol index

$U \quad$ velocity in $x$ direction $(\mathrm{m} / \mathrm{s})$

$V \quad$ velocity in $y$ direction $(\mathrm{m} / \mathrm{s})$

\section{Greek Symbols}

$\alpha \quad$ spectral modes

$\alpha^{*} \quad$ stochastic output variable

$\mu_{t} \quad$ turbulent (eddy) viscosity $(\mathrm{kg} /(\mathrm{m} \cdot \mathrm{s}))$

$\xi \quad$ standard random variable variable(s)

$\vec{\xi}_{a} \quad$ standard aleatory random

$\vec{\xi}_{e} \quad$ standard epistemic random variable(s)

$\tau \quad$ shear stress $\left(\mathrm{N} / \mathrm{m}^{2}\right)$

$\Psi \quad$ random basis function
Subscript
ref reference condition
$\mathrm{w} \quad$ wall condition
$\infty \quad$ free stream condition

of counter-rotating vortices. These vortical structures then move away from the orifice under their own self-induced velocity. In the presence of a cross flow, these vortex pairs convect downstream entraining fluid from the free stream, resulting in favorable local displacement of the streamlines and pressure distribution changes at these regions. In recent years there have been a number of experimental and numerical investigations of the pulsating synthetic jets. A comprehensive list of these studies can be found in a review paper by Glezer and Amitay [1].

Among all the investigations in the flow phenomena involved in a synthetic jet application, computational fluid dynamics (CFD) simulations are becoming more and more important with the aim of accurately predicting the flow field quantities and being able to perform robust and reliable designs. In order to assess the state-of-the-art CFD modeling of synthetic jet flows, a validation workshop [2] (referred as "CFDVAL2004" in the rest of this paper) was held in 2004 from which several synthetic jet configurations were selected as test cases. Summary of the workshop results and conclusions can be found in Rumsey et al. [3]. One of the conclusions was that, due to the unknown uncertainties in the modeling of the configuration, the CFD results failed to consistently agree with the experiments [4]. The timedependent flow field quantities, such as phase-averaged velocities, as well as the long-time averaged quantities can be affected by the uncertainties in the initial conditions, the unsteady boundary conditions, and physical models (e.g., turbulence model) used in simulations. In addition, the flow field also can be affected by the variations in operating conditions such as the main stream velocity in the presence of cross flow. All these uncertainties associated with the CFD modeling of synthetic jet actuators motivate an uncertainty quantification (UQ) study to assess the accuracy of the results.

The objective of this study is to introduce and demonstrate an efficient methodology for the quantification of uncertainties and global nonlinear sensitivity analysis in CFD modeling of synthetic jet actuators, which include both inherent (aleatory) and model-form (epistemic) uncertainty sources. One unique aspect of the current study is to consider both aleatory and epistemic input uncertainties and quantify the contribution of each uncertainty source to the overall uncertainty in a selected output quantity. Although we focus on the uncertainty quantification of a synthetic jet problem in the current study, the mixed uncertainty quantification approach demonstrated in this study can also be applied to other computational fluid dynamics problems with inherent and model-form input uncertainties (see Refs. [5-7] for applications in high-speed flow problems).

The second-order probability theory can be used to propagate mixed (aleatory and epistemic) uncertainties through a simulation code. However, numerical computations can be intensive for this particular method due to the use of nested loops, especially if the simulation code is expensive to run (such as a high-fidelity CFD code). To address this issue, a more efficient approach to second-order probability is described in this work. In particular, a stochastic response surface which is obtained using a nonintrusive polynomial chaos (NIPC) method (Hosder et al. [8]) is utilized 
in the second-order probability framework. The stochastic response surface is a surrogate model for the original simulation code, and is computationally less expensive to evaluate. Therefore, the utilization of the stochastic response surface, formulated with NIPC methods, enables the propagation of mixed uncertainties through the simulation code with much less computational cost compared to the expense of a traditional direct sampling approach (e.g., Monte Carlo methods).

In the current work, a synthetic jet issued into a cross flow over a two-dimensional wall-mounted hump-shaped body (Case 3 of CFDVAL2004) is selected as the CFD modeling problem with both epistemic and aleatory uncertain inputs. Three uncertain variables are considered: turbulent (eddy) viscosity coefficient obtained from the turbulence model (epistemic or model-form uncertainty), free stream velocity (aleatory), and the frequency used in the unsteady velocity-inlet boundary condition imposed at the bottom of the synthetic jet actuator cavity (aleatory uncertainty), which represent the variations in the frequency of the oscillating piston. Both aleatory uncertain inputs are described with uniform probability distributions, whereas the uncertainty in the turbulent viscosity is represented with an interval due to its epistemic nature. The quantities of interest for uncertainty quantification in the CFD simulations include the long-time averaged separation bubble characteristics, pressure and skin friction coefficients, as well as the phaseaveraged $x$-velocity distributions at selected locations. A previous study on another synthetic jet case (Case 1 of CFDVAL2004) by Adya et al. [9] conducted the UQ analysis with two aleatory uncertainty variables (amplitude and frequency of actuation). The synthetic jet configuration studied in the current work extends the flow phenomenon to the presence of a cross flow, with a more comprehensive treatment of uncertainty sources including the turbulence model (epistemic uncertainty).

The paper is organized as follows: in the next section, the mixed uncertainty quantification approach will be described. The computational model and stochastic problem with uncertain inputs will be described in Section 3 . In Section 4, the uncertainty quantification results will be outlined and discussed. The conclusions will be given in Section 5.

\section{UNCERTAINTY QUANTIFICATION APPROACH}

\subsection{Types of Uncertainties in Computational Simulations}

Generally, there are two types of uncertainty in a computational simulation: (1) aleatory uncertainty and (2) epistemic uncertainty. A detailed description of these uncertainties can be found in Oberkampf et al. [10].

Aleatory uncertainty, or inherent uncertainty, originates from the random nature of a physical system and thus can be mathematically represented by a probability density function (PDF) if knowledge is available to estimate the distribution type (e.g., uniform, normal, etc.). Such knowledge can be from the substantial experimental data, statistical study of the survey, etc. The uncertainty in free stream velocity or geometry can be treated as examples of aleatory uncertainties in a stochastic aerodynamics problem.

Epistemic uncertainty, also referred as model-form uncertainty, is due to ignorance, lack of knowledge, or incomplete information of some characteristics from a nondeterministic system. By this feature, an increase in knowledge or understanding of a system can lead to a decrease in the epistemic uncertainty. Similar to aleatory uncertainty, epistemic uncertainty can be also modeled with probabilistic approach. However, studies [11] have shown that this may cause inaccurate predictions in the amount of uncertainty in the responses. Another method to treat the epistemic uncertain variables is to use intervals by giving the lower and upper bounds with the information from limited experimental data, expert judgment, or an empirical model.

\subsection{Mixed (Aleatory-Epistemic) Uncertainty Propagation}

\subsubsection{Second-Order Probability}

In the current work, second-order probability $[12,13]$ is employed to propagate mixed aleatory and epistemic uncertainty through CFD simulations. As described in Bettis et al. [14], second-order probability (Fig. 1) approach uses an outer loop where a specific value of the epistemic variable is selected and an inner loop where any traditional aleatory uncertainty quantification method can be performed for uncertainty analysis at that specific value of the epistemic 


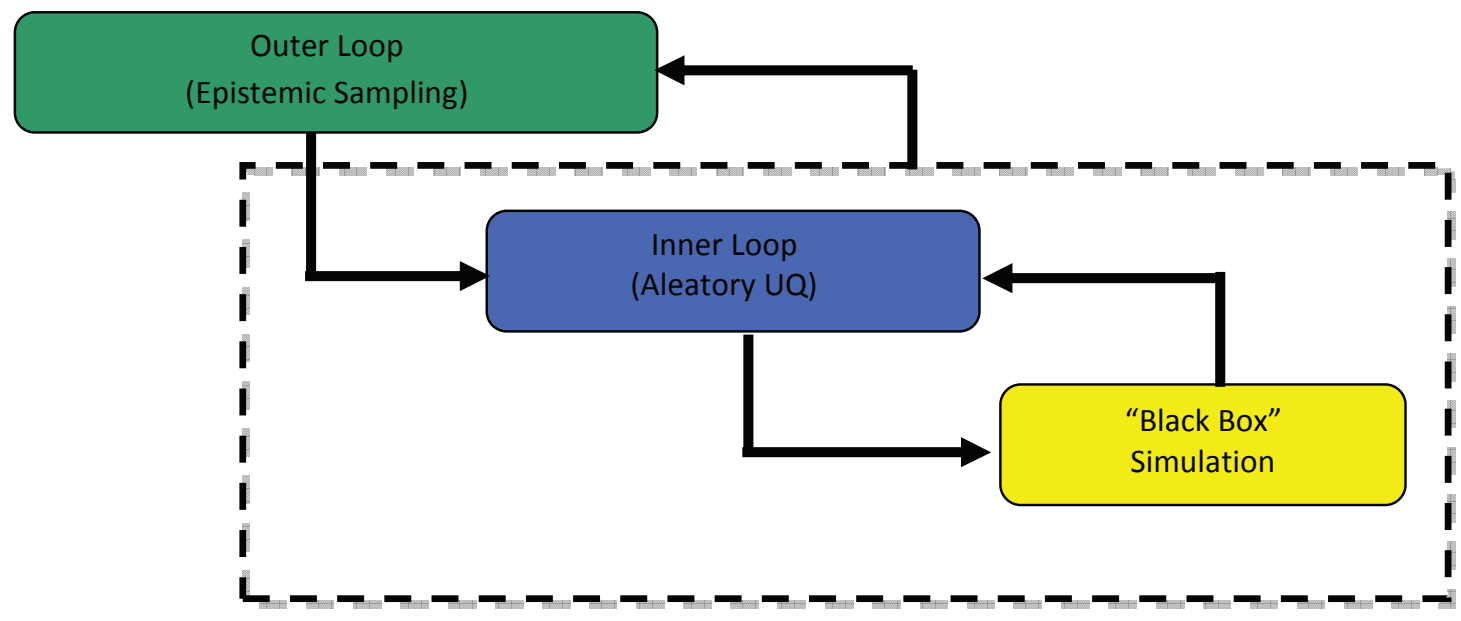

FIG. 1: Schematic of second-order probability (Taken from Bettis et al. [14]).

variable. Each iteration of the outer loop will produce a cumulative distribution function (CDF) based on the aleatory uncertainty analysis in the inner loop. So the Second-Order Probability approach will produce a family of CDFs and give intervals of the output at different probability levels. Since the epistemic and aleatory variables are treated in different loops, it is easy to separate and identify each of them from the output horsetail plots. However, this method can be relatively computationally expensive due to the two sampling loops especially when traditional sampling approach such as Monte Carlo is used.

In this study, first a stochastic response surface (function of both epistemic and aleatory variables) for the output quantity of interest is obtained using a quadrature-based NIPC method (described below). Then the second-order probability is employed by sampling the epistemic uncertain variables in the outer loop, sampling the aleatory uncertain variables in the inner loop (with fixed values of epistemic uncertain variables), and finally evaluating the output from the NIPC response surface approximation.

\subsubsection{Quadrature-Based Nonintrusive Polynomial Chaos}

In the current study, the quadrature-based nonintrusive polynomial chaos (NIPC) is employed which is derived from the polynomial chaos theory based on the spectral representation of the uncertainty. As an important aspect of spectral representation of uncertainty, one can decompose a random function or variable into separable deterministic and stochastic components as shown in Eq. (1) where $\alpha^{*}$ can be any random variable of interest such as long-time averaged or phase-averaged velocity, pressure, or skin friction coefficient in a stochastic fluid dynamics problem.

$$
\alpha^{*}(t, \vec{x}, \vec{\xi}) \approx \sum_{j=0}^{P} \alpha_{j}(t, \vec{x}) \Psi_{j}(\vec{\xi})
$$

In the equation above, $\alpha_{j}(t, \vec{x})$ is the deterministic component and $\Psi_{j}(\vec{\xi})$ is the random basis function corresponding to the $j$ th mode. Here $\alpha^{*}$ is assumed to be a function of the independent deterministic variable vector $(t, \vec{x})$ and the $n$-dimensional random variable vector $\vec{\xi}=\left(\xi_{1}, \ldots, \xi_{n}\right)$, which can include both aleatory and epistemic uncertain variables. In theory, the polynomial chaos expansion (PCE) given by Eq. (1) should have infinite number of terms. However, in practice a discrete summation is taken over a finite number of output modes. For a total order expansion, the number of output modes $\left(N_{t}\right)$ is given by

$$
N_{t}=P+1=\frac{(n+p) !}{n ! p !},
$$


which is a function of the order of PCE $(p)$ and the number of random dimensions $(n)$. Ideally, the basis function takes the form of multi-dimensional Hermite polynomial to span the $n$-dimensional random space when the input uncertainty is Gaussian (unbounded), which was first introduced by Wiener [15] in his original work of polynomial chaos. To extend the application of the polynomial chaos theory to the propagation of continuous nonnormal input uncertainties, Xiu and Karniadakis [16] used a set of polynomials known as the Askey scheme to obtain the Wiener-Askey Generalized Polynomial Chaos. Among the polynomials included in the Askey scheme, the Legendre and Laguerre polynomials are optimal basis functions for bounded (uniform) and semi-bounded (exponential) input uncertainty distributions respectively in terms of the convergence of the statistics. For problems having more than one uncertainty variable, the multivariate basis functions can be obtained from the product of univariate orthogonal polynomials (see Eldred et al. [17]). If the probability distribution of each random variable is different, the optimal multivariate basis functions can be again obtained from the product of univariate orthogonal polynomials employing the optimal univariate polynomial at each random dimension. In this approach, it is required that the input uncertainties are independent standard random variables, which also allows the calculation of the multivariate weight functions by taking the product of univariate weight functions associated with the probability distribution at each random dimension. More detailed information on PCE can be found in Walters and Huyse [18], Najm [19], and Hosder and Walters [8].

Generally there are intrusive and nonintrusive approaches to model the uncertainty propagation in computational simulations via PCE. In the intrusive approach, all dependent variables and random parameters in the governing equations are replaced with their PCEs. Taking the inner product of the equations, or projecting each equation onto $j$ th basis, yields $(P+1)$ times the number of deterministic equations which can be solved by the same numerical methods applied to the original deterministic system (computational simulation). Although straightforward in theory, an intrusive formulation for complicated problems can be relatively difficult, expensive, and time-consuming to implement. To overcome such inconveniences associated with the intrusive approach, nonintrusive polynomial chaos formulations are considered for uncertainty propagation in this study.

The quadrature-based NIPC method employed in this paper uses spectral projection to find the polynomial coefficients $\alpha_{k}=\alpha_{k}(t, \vec{x})$ in Eq. (1). Projecting Eq. (1) onto the $k$ th basis yields

$$
\left\langle\alpha^{*}(t, \vec{x}, \vec{\xi}), \Psi_{k}(\vec{\xi})\right\rangle=\left\langle\sum_{j=0}^{P} \alpha_{j}(t, \vec{x}) \Psi_{j}(\vec{\xi}) \Psi_{k}(\vec{\xi})\right\rangle
$$

then, from the virtue of orthogonality,

$$
\left\langle\alpha^{*}(t, \vec{x}, \vec{\xi}), \Psi_{k}(\vec{\xi})\right\rangle=\alpha_{k}(t, \vec{x})\left\langle\Psi_{k}^{2}(\vec{\xi})\right\rangle,
$$

which leads to

$$
\alpha_{k}(t, \vec{x})=\frac{\left\langle\alpha^{*}(t, \vec{x}, \vec{\xi}), \Psi_{k}(\vec{\xi})\right\rangle}{\left\langle\Psi_{k}^{2}(\vec{\xi})\right\rangle}=\frac{1}{\left\langle\Psi_{k}^{2}(\vec{\xi})\right\rangle} \int_{R} \alpha^{*}(t, \vec{x}, \vec{\xi}) \Psi_{k}(\vec{\xi}) p(\vec{\xi}) d \vec{\xi} .
$$

The objective of the spectral projection method is to predict the polynomial coefficients by evaluating the numerator $\left(\left\langle\alpha^{*}(t, \vec{x}, \vec{\xi}), \Psi_{k}(\vec{\xi})\right\rangle\right)$ in Eq. (5), while the denominator $\left(\left\langle\Psi_{k}^{2}(\vec{\xi})\right\rangle\right)$ can be computed analytically for multivariate orthogonal polynomials.

In the quadrature-based nonintrusive approach, the multi-dimensional integral in the numerator of Eq. (5) is evaluated with numerical quadrature [17] in the support range $(R)$ where input uncertain variables are defined. For the integration of one-dimensional problems, the straightforward approach is to use Gaussian quadrature points, which are zeros of the orthogonal polynomials that are optimal for the given input uncertainty distribution (e.g., Gauss-Hermite, Gauss-Legendre, and Gauss-Laguerre points for normal, uniform, and exponential distributions, respectively). The extension of this approach to multi-dimensional problems can be achieved via tensor product of one-dimensional quadrature formula. In one-dimensional problems, a Gauss quadrature formula of $n_{p}$ points will exactly evaluate a polynomial of degree $\left(2 n_{p}-1\right)$ or less and the polynomial degree of the product of the function approximation and the basis in the integrand of the numerator in Eq. (5) will be $2 p$ for the evaluation of the coefficient of the highest degree

Volume 4, Number 6, 2014 
if the degree of the PCE is chosen as $p$. Therefore, the minimum number of quadrature points required to exactly evaluate the integral will be $(p+1)$. Once the number of uncertain variables and degree of PCE are determined, the quadrature points are fixed. This can be a potential disadvantage of quadrature-based NIPC if the CFD evaluations at some quadrature points are not converged. For stochastic problems with relatively small number of input uncertain variables (i.e., $n \leq 4$ ), this approach will be computationally efficient compared to a typical Monte Carlo approach. However, for multi-dimensional problems with large number of uncertain variables, the computational expense may become significant due to its exponential growth with the number of random dimensions, since the required number of deterministic function evaluations will be $(p+1)^{n}$ for a stochastic problem with $n$ random variables having the same degree of PCE $(p)$ in each dimension. The computational expense of propagating mixed input uncertainties can be high even if the number of aleatory and epistemic uncertain variables is not large when the "deterministic function evaluation" is actually a CFD simulation. Therefore constructing and evaluating a stochastic (polynomial chaos) response surface will significantly reduce the required number of deterministic CFD simulations for the propagation of mixed uncertainties.

\subsubsection{Second-Order Probability with Stochastic Response Surface}

As described previously, the current study utilizes second-order probability approach to propagate the mixed aleatoryepistemic uncertainties. In this approach, the stochastic response (e.g., the long-time averaged separation bubble size) is represented with a PCE as a function of both aleatory and epistemic uncertain variables. The optimal basis functions are used for the aleatory variables while Legendre polynomials are used for the epistemic variables. Note that the use of Legendre polynomials should not imply uniform probability distributions of the epistemic uncertain variables. This choice is made due to the bounded nature of epistemic uncertain variables considered in this study. Once the stochastic response surface is constructed, the stochastic responses can be evaluated for a large number of samples randomly produced based on the probability distributions of the aleatory input uncertainties (inner loop of second-order probability) with fixed values of epistemic uncertain variables. Each iteration in the outer loop will produce a single cumulative distribution function (CDF). By repeating the inner loop for a large number of epistemic uncertain variables sampled from their corresponding intervals (outer loop of second-order probability), a population of cumulative distribution functions can be obtained (and presented as a "p-box" or "horsetail" plot), thus the bounds of the stochastic response at different probability levels (e.g., 2.5\% and 97.5\%) can be calculated. Figure 2 gives an example of a conservative calculation of $95 \%$ confidence interval (CI) obtained as the difference between the upper limit of $97.5 \%$ probability level and the lower limit of the $2.5 \%$ probability level, which will be used in the current study.

Due to the analytical nature (polynomials) of the stochastic response surface, the described procedure will be computationally efficient, especially compared to the direct Monte Carlo sampling which requires a large number of deterministic CFD simulations. Figure 3 shows the flowchart of the entire procedure of mixed aleatory-epistemic uncertainty analysis employed in this study.

\subsubsection{Global Sensitivity Analysis with Sobol Indices}

In a system where multiple uncertain variables are present, it is often useful to demonstrate and rank the relative importance of each input uncertain variable to the overall output quantity of interest using a global sensitivity analysis approach. In the current study, Sobol [20] indices are used to perform this analysis.

Once the PCE for an output uncertain variable is formed using Eq. (5), Sobol indices can be derived via Sobol decomposition, which is a variance-based global sensitivity analysis method. First, the total variance $(D)$ can be written in terms of the PCE:

$$
D=\sum_{j=1}^{P} \alpha_{j}^{2}(t, \vec{x})\left\langle\Psi_{j}^{2}(\vec{\xi})\right\rangle .
$$

Then, as shown by Sudret [21] and Crestaux et al. [22], the total variance can be decomposed as

$$
D=\sum_{i=1}^{i=n} D_{i}+\sum_{1 \leq i<j \leq n}^{i=n-1} D_{i, j}+\sum_{1 \leq i<j<k \leq n}^{i=n-2} D_{i, j, k}+\cdots+D_{1,2, \ldots, n},
$$




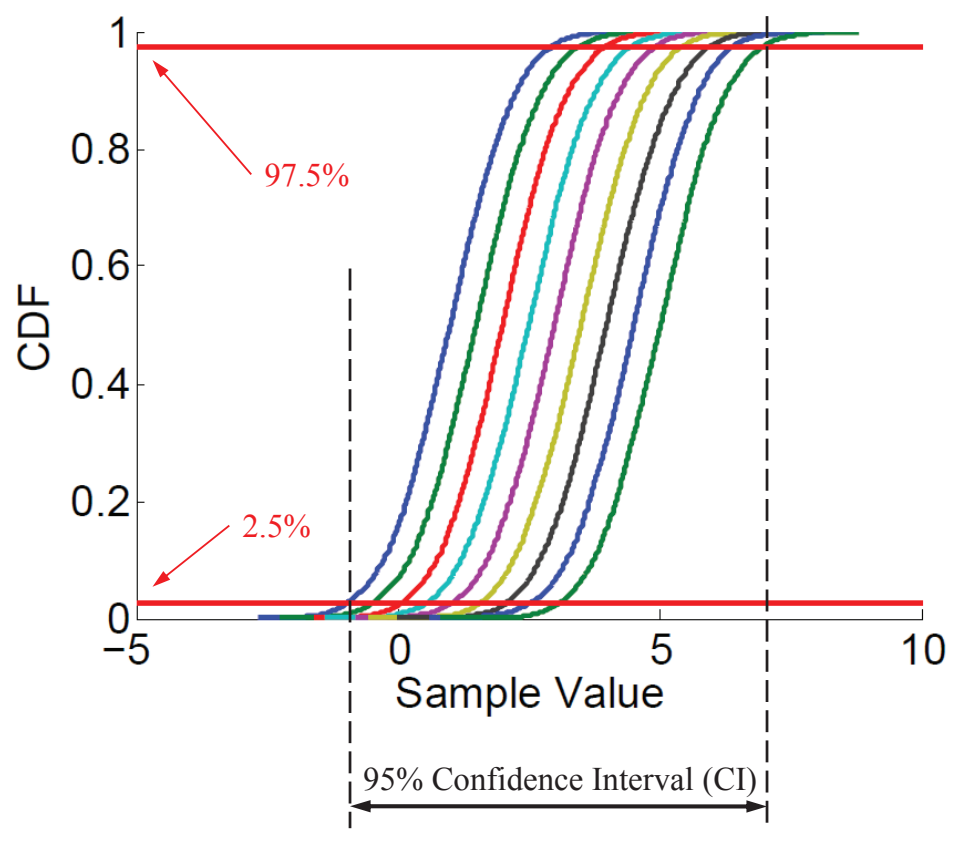

FIG. 2: A typical probability-box (p-box) used in the representation of mixed uncertainty output and conservative calculation of $95 \%$ confidence interval.

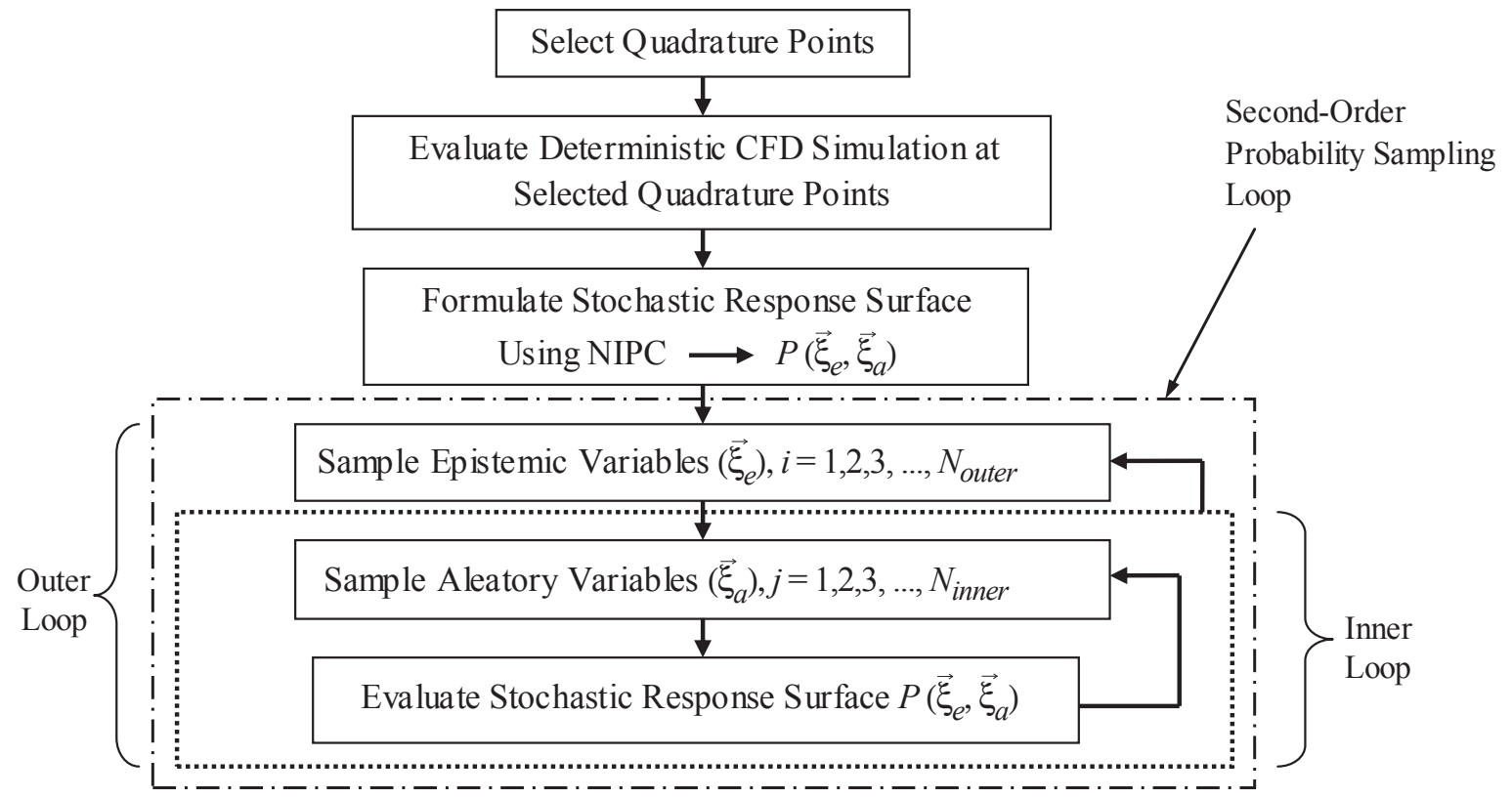

FIG. 3: Flowchart showing the procedure of propagating mixed aleatory-epistemic uncertainty with second-order probability and quadrature-based NIPC.

where the partial variances $\left(D_{i_{1}, \ldots, i_{s}}\right)$ are given by

$$
D_{i_{1}, \ldots, i_{s}}=\sum_{\beta \in\left\{i_{1}, \ldots, i_{s}\right\}} \alpha_{\beta}^{2}\left\langle\Psi_{\beta}^{2}(\vec{\xi})\right\rangle, \quad 1 \leq i_{1}<\ldots<i_{s} \leq n .
$$


Then the Sobol indices $\left(S_{i_{1} \cdots i_{s}}\right)$ are defined as

$$
S_{i_{1} \ldots i_{s}}=\frac{D_{i_{1}, \ldots, i_{s}}}{D}
$$

which satisfy the following equation:

$$
\sum_{i=1}^{i=n} S_{i}+\sum_{1 \leq i<j \leq n}^{i=n-1} S_{i, j}+\sum_{1 \leq i<j<k \leq n}^{i=n-2} S_{i, j, k}+\cdots+S_{1,2, \ldots, n}=1.0 .
$$

The Sobol indices provide a sensitivity measure due to individual contribution from each input uncertain variable $\left(S_{i}\right)$, as well as the mixed contributions $\left(\left\{S_{i, j}\right\},\left\{S_{i, j, k}\right\}, \ldots\right)$. As shown by Sudret [21] and Ghaffari et al. [23], the total (combined) effect $\left(S_{T_{i}}\right.$ ) of an input parameter $i$ is defined as the summation of the partial Sobol indices that include the particular parameter

$$
S_{T_{i}}=\sum_{L_{i}} \frac{D_{i_{1}, \ldots, i_{s}}}{D} ; \quad L_{i}=\left\{\left(i_{1}, \ldots, i_{s}\right): \exists k, 1 \leq k \leq s, i_{k}=i\right\} .
$$

For example, with $n=3$, the total contribution to the overall variance from the first uncertain variable $(i=1)$ can be written as

$$
S_{T_{1}}=S_{1}+S_{1,2}+S_{1,3}+S_{1,2,3}
$$

From these formulations, it can be seen that the Sobol indices can be used to provide a relative ranking of each input uncertainty to the overall variation in the output with the consideration of nonlinear correlation between input variables and output quantities of interest. One of the goals of the current work is to calculate Sobol indices with the PCE and then use them to rank the relative importance of each input uncertain variable to a specific output quantity of interest.

\section{COMPUTATIONAL MODEL}

\subsection{CFD Simulations}

\subsubsection{Physical Model and Geometry}

The synthetic jet configuration studied in this paper is flow over a two-dimensional wall-mounted hump-shaped body which was labeled as "hump model (Case 3)" in CFDVAL2004 workshop [2].

Figure 4 shows the experimental configuration [2] of the hump model which was mounted between two glass endplate frames. The width of the tunnel test section was 28" and the nominal test section height was 15.032". As the workshop indicates, this experiment was nominally two-dimensional except the side wall effects near the endplates.

The characteristic reference length of the model is defined as the length of the bump on the wall which is 16.536". The model itself is 23" wide between the endplates at both sides and 2.116" high at its maximum thickness point. All the experiment test flows were considered under the free stream conditions of Mach $=0.1$ at a Reynolds number of $9.36 \times 10^{5}$. The model experienced a fully developed turbulent boundary layer during the test, which separated over the concave section in the aft part of the hump body. A slot opening was located at approximately $65 \%$ chord station, extending across the entire span of the hump. In the oscillatory part of the experiment, the two-dimensional oscillatory blowing was achieved by means of a rigid piston spanning the model with a frequency of $138.5 \mathrm{~Hz}$. More detailed test conditions are documented on the website of CFDVAL2004 [2].

\subsubsection{Numerical Scheme and Computational Grid}

The commercial CFD software, ANSYS FLUENT 12 [24], was used for the simulation of the flow field. The unsteady Reynolds-averaged Navier-Stokes (RANS) equations coupled with Spalart-Allmaras [25] turbulence model 


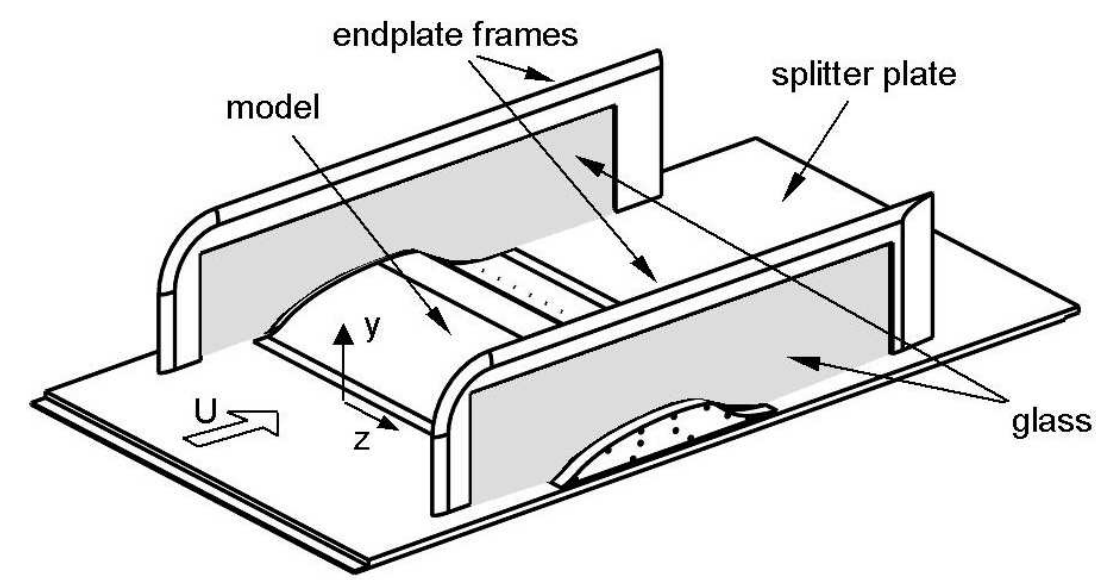

FIG. 4: Experimental configuration (taken from CFDVAL2004 [2]).

were solved to compute the unsteady, turbulent, two-dimensional flow field including the cavity and main flow region. Periodic solutions were obtained to calculate the phase-averaged and long-time averaged quantities in the flow field. A second-order accurate implicit time-integration scheme was used to advance the solution in time. The inviscid fluxes were approximated with a second-order upwind scheme in space and the viscous terms were approximated with second-order central differencing.

The grid employed in this paper was labeled as "STRUCTURED 2D GRID \#4" on the workshop website (210,060 grid points total), where top wall shape was adjusted to approximately account for the side plate blockage effect. In this grid, the computational domain extended upstream to -6.39 chord length which yields a "run" long enough to get the approximate boundary layer thickness matching experimental data. The internal slot and cavity were also included in the grid. Figure 5 shows the local zoom-in view of the grid near the slot [2].

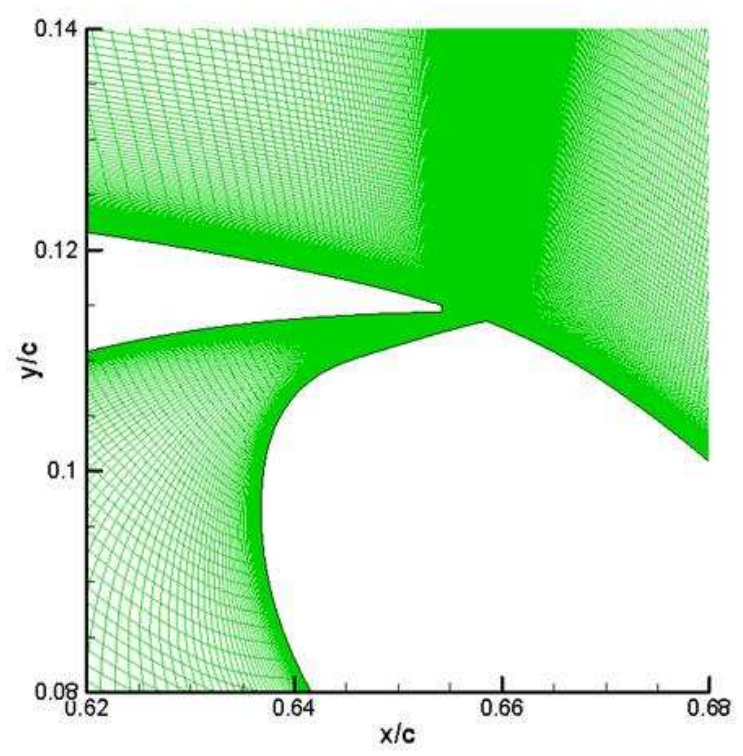

(a)

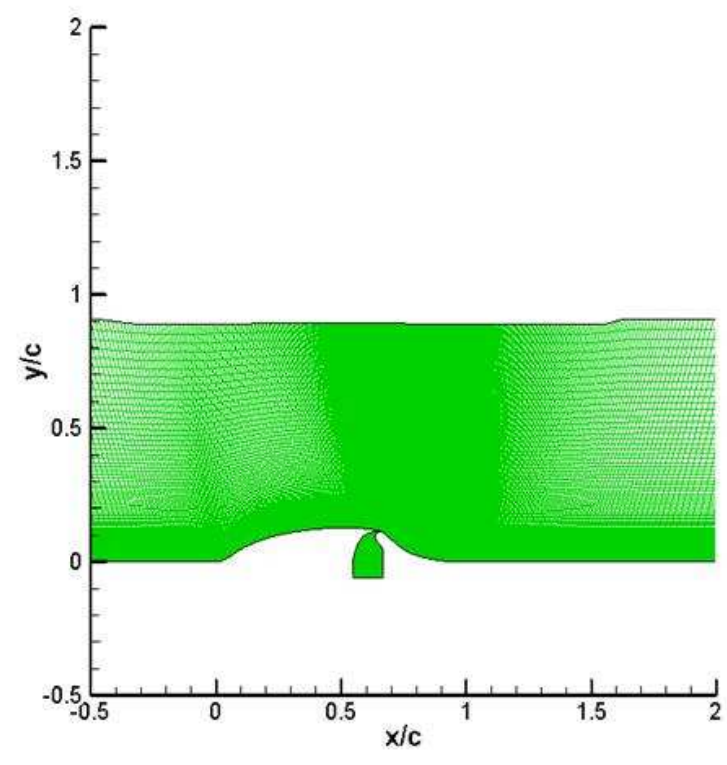

(b)

FIG. 5: (a) Zoom-in view of slot region grid; (b) Main flow domain. 
To get the time-accurate solution, 360 time steps per period were used with 20 inner iterations per time step. All the simulation results presented are taken from cycles when periodicity was obtained. Phase-averaged and longtime averaged data were calculated to compare the results of CFD simulations with available experimental data. The reference phase was defined as the maximum blowing occurring at a phase angle of $170^{\circ}$ and maximum suction at a phase angle of $350^{\circ}$.

\subsubsection{Boundary Conditions}

At the floor and surfaces, as well as the inner side of the cavity, solid nonslip wall conditions were applied. At the location $x / c=-6.39$ where velocity-inlet boundary condition was applied, uniform velocity profile was used to get a naturally developed full-turbulent boundary layer so that it reaches the approximate boundary layer thickness as experimental data measured at the location of $x / c=-2.14$. At the downstream boundary, pressure-outlet boundary type was applied with the pressure $p / p_{\text {ref }}=0.99962$, where $p_{\text {ref }}$ was the free stream reference pressure. At top wall of the tunnel, inviscid wall condition was applied for the consideration of side plate blockage effect. The boundary condition at the bottom of the cavity was set as velocity-inlet where the components of the velocity were given as follows:

$$
\begin{gathered}
U=0, \\
V=A_{0} \cos (2 \pi f t),
\end{gathered}
$$

where the amplitude $A_{0}$ was picked to match the peak velocity out of slot during blowing part of cycle in the experiment [26]. Figure 6 shows the schematics of the boundary conditions applied in the CFD simulation.

\subsection{Description of the Stochastic Problem}

For this study, the free stream velocity $\left(U_{\infty}\right)$ and frequency in the unsteady velocity-inlet boundary condition $(f)$ imposed to the cavity bottom were modeled as aleatory uniformly distributed uncertain variables with a coefficient of variance $(\mathrm{CoV})$ of $10 \%$ from their baseline values. The turbulent viscosity coefficient within the Spalart-Allmaras (S-A) turbulence model [25] was treated as a source of epistemic uncertainty through the introduction of a factor $K$ as shown below:

$$
\mu_{t}=K \mu_{t_{S A}},
$$

where $\mu_{t_{S A}}$ was the turbulent viscosity originally calculated in the S-A model and then scaled by factor $K$ as the turbulent viscosity used in the whole computational domain in the CFD simulations. The range of this factor $K$ was chosen based on the turbulent viscosities calculated from different turbulence models (i.e., standard $k-\epsilon$, standard $k-\omega$

Top wall contoured to approximately account for side plate blockage effect
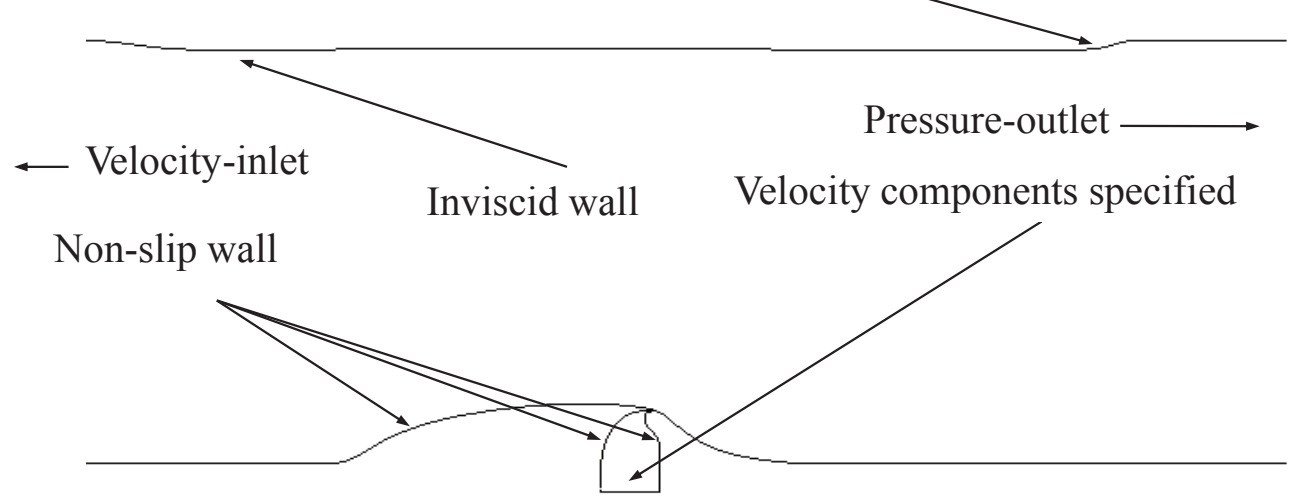

FIG. 6: Overview of boundary conditions applied in CFD simulation. 
and SST $k-\omega$ ) [24] for the baseline case to reflect the uncertainty due to the use of different turbulence models. All other parameters in the CFD simulations were kept constant at their baseline values. An overview of the ranges of the uncertain parameters considered in this study is shown in Table 1.

\section{UNCERTAINTY QUANTIFICATION RESULTS}

In this study, long-time averaged separation bubble size, pressure and skin friction coefficients and phase averaged $\mathrm{x}$-velocity distributions at several locations were selected as the output quantities of interest to perform the uncertainty quantification analysis.

\subsection{Uncertainty Quantification in Long-time Averaged Bubble Size}

Figure 7 is a sample CFD result from the CFDVAL2004 workshop showing the main flow structure near the separation bubble region. In this study, the long-time averaged separation bubble size (calculated with separation and reattachment locations) was chosen to represent the bubble characteristics. The preliminary results of the current study, as well as Rumsey [26], showed that the location of separation was relatively insensitive to the parameters considered. The reattachment location was found to have a larger variance. The separation bubble size was the difference between separation and reattachment locations and obtained by locating the points on the wall where the long-time averaged skin-friction values were equal to zero.

\subsubsection{Results with Pure Aleatory Uncertainty Assumption}

Before mixed aleatory-epistemic uncertainty quantification, the analysis with pure aleatory uncertainty assumption was conducted where all three uncertain input variables were treated as aleatory with uniform uncertainty distributions with the bounds given in the previous section. The CDFs obtained from different degrees of PCE were compared. The quadrature-based NIPC method described in the previous section was used to construct the response surface as a

TABLE 1: Uncertainty ranges for parameters used in CFD simulations

\begin{tabular}{|ccc|}
\hline \hline Uncertain parameter & Uncertainty type & Uncertainty range \\
\hline \hline$U_{\infty}$ & Aleatory (uniform) & {$[31.14,38.06] \mathrm{m} / \mathrm{s}$} \\
$f$ & Aleatory (uniform) & {$[124.65,152.35] \mathrm{Hz}$} \\
$K$ & Epistemic & {$[0.5,2.0]$} \\
\hline \hline
\end{tabular}

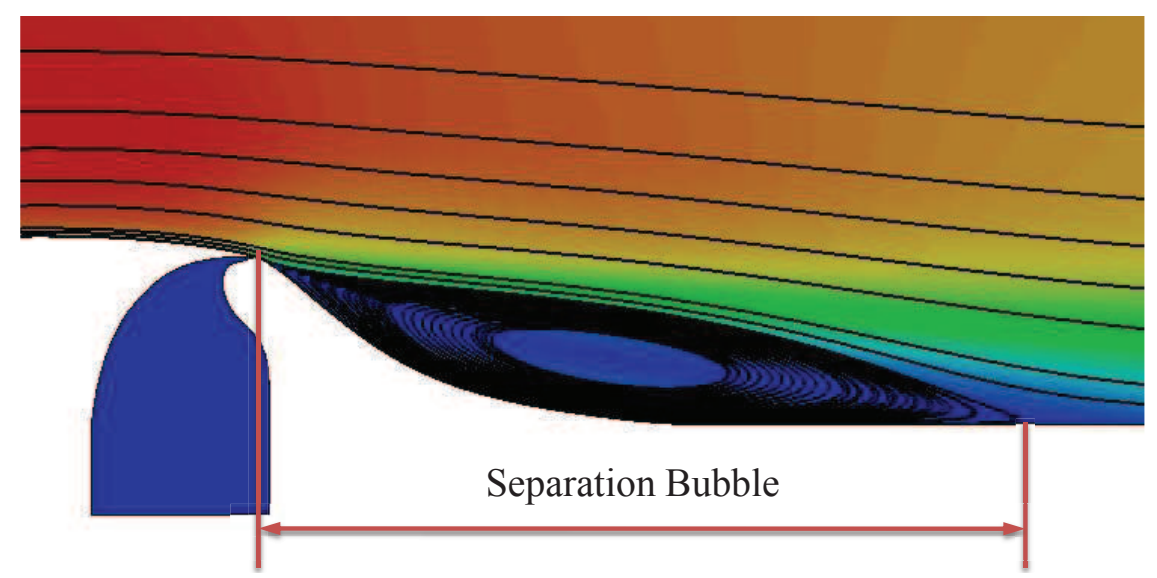

FIG. 7: A sample CFD result from CFDVAL2004 [2]. 
function of all three uncertain input variables. It is important to ensure that the order of PCE is high enough to capture the nonlinear relations between input and output quantities of interest. Therefore, a degree convergence study was performed where the PCE order was increased up to 4 and the response surface was constructed and evaluated at each order. Figure 8 gives an example of the CDF plots to show the degree convergence of the PCE with respect to longtime averaged separation bubble size; 10,000 random samples were selected from the stochastic response surface to evaluate the $\mathrm{CDF}$ at each polynomial degree. The figure shows that there is no obvious difference in the CDFs between third and fourth orders of PCE. Thus it can be concluded that the response surface obtained via the quadrature-based NIPC converged at the third order PCE. The results presented in this paper are from the fourth order PCE.

\subsubsection{Results with Mixed (Aleatory-Epistemic) Uncertainty Assumption}

For the mixed (aleatory-epistemic) uncertainty quantification, second-order probability approach described previously was used with the same response surface obtained for pure aleatory uncertainty assumption (fourth order PCE). Random samples from the specified bounds (Table 1) were utilized for the epistemic uncertain variable in the outer loop while in the inner loop, for each specific value of the epistemic uncertain variable, samples of aleatory uncertain variables based on the uniform probability distributions were utilized to evaluate the stochastic response surface. Two sets of samples were selected to check the sample size independence. The first set took 100 samples in the outer (epistemic) loop and 1000 samples in the inner (aleatory) loop; the second set took 1000 and 10,000 samples in the outer and inner loops, respectively. The CDFs produced are shown in Fig. 9. For each set of samples, it is obvious that at a particular probability level, the variation in the long-time averaged separation bubble size is due to the epistemic uncertain input ( $K$ factor), which is represented by the interval bounded by the minimum and maximum values obtained from the CDFs at the same probability level. The width of the interval is nearly constant at each probability level. The overall agreement of the horsetail plots obtained from the two sets of samples also shows the sample size independence in the second-order probability framework. Thus in the following part of this study, 100 samples for the epistemic loop and 1000 samples for the aleatory loop were utilized considering the computational expenses.

\subsubsection{Global Sensitivity Analysis with Sobol Indices}

In order to have a relative ranking of the importance of each input uncertain variable to the overall output uncertainty in the long-time averaged separation bubble size, global sensitivity analysis with Sobol indices was conducted to

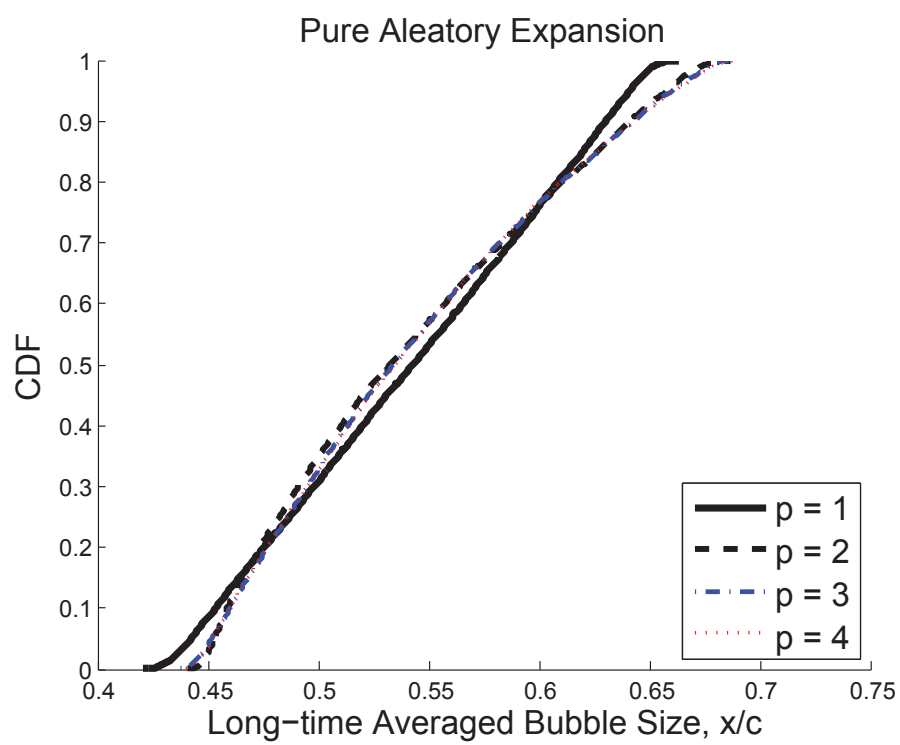

FIG. 8: PCE degree convergence check of long-time averaged bubble size. 

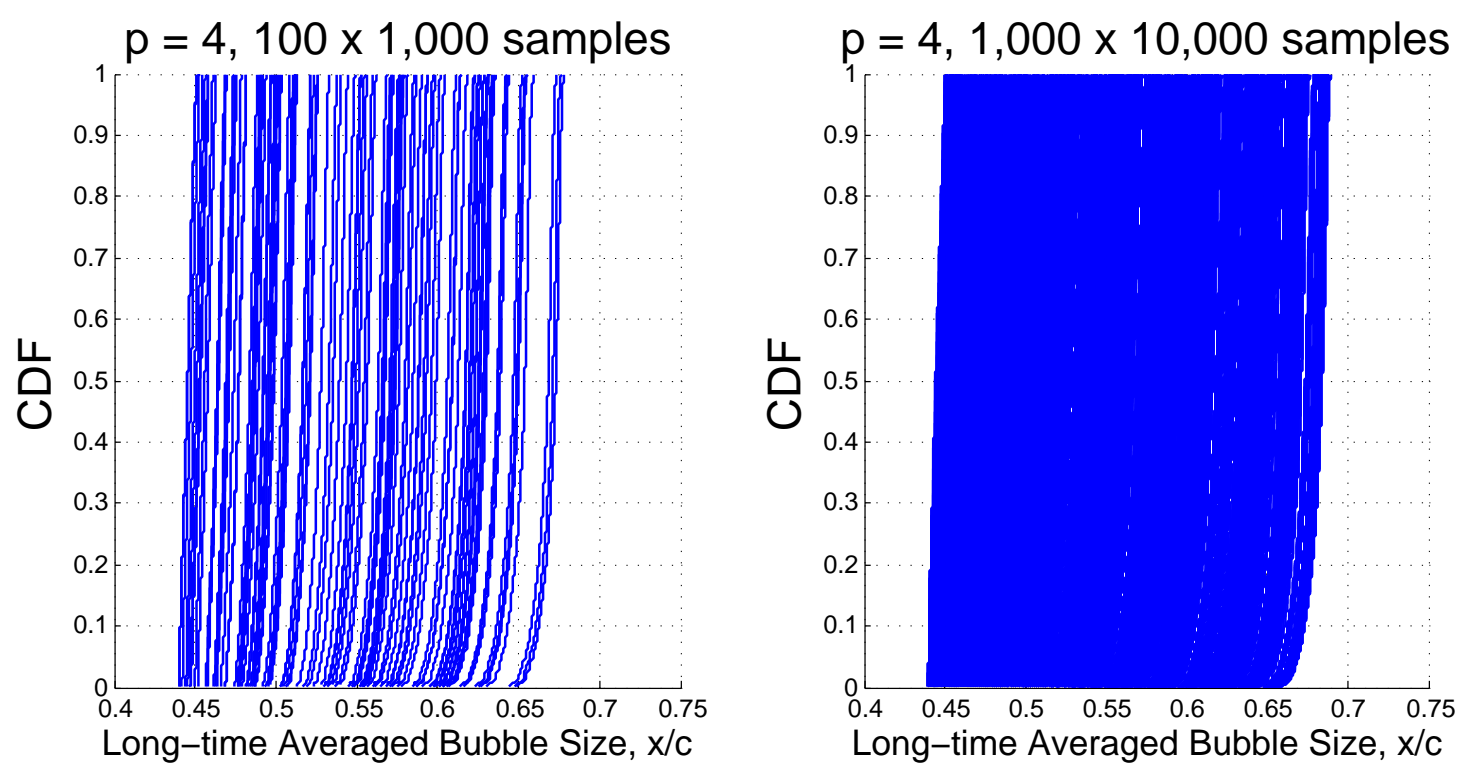

FIG. 9: P-box plots for long-time averaged bubble size.

quantitatively account for the nonlinear dependencies between input and output uncertainties. These indices obtained from the same order of PCE (fourth) in the previous sections are shown in Table 2. The results show that the epistemic input uncertain variable $K$ factor, thus the turbulent eddy viscosity, has a dominant influence on the output uncertainty in the long-time averaged bubble size while the two aleatory input uncertain variables have much less contributions to the output uncertainty. This is consistent with the observation from the horsetail plots shown in Fig. 9. This result also agrees qualitatively well with a similar study by Rumsey [26]. Furthermore, the results also indicate that the combined contributions of different uncertainty sources (e.g., $S_{1,2}$ ) are very small when comparing the total indices (e.g., $S_{T_{1}}$ ) with their corresponding individual (noncombined) indices (e.g., $S_{1}$ ).

\subsection{Uncertainty Quantification in Long-Time Averaged Pressure and Skin Friction Coefficients}

\subsubsection{Results with Pure Aleatory Uncertainty Assumption}

Before the mixed uncertainty quantification, analysis with pure aleatory uncertainty assumption was conducted where all three uncertain input variables were treated as aleatory with uniform uncertainty distributions with the bounds given in the previous section. The CDFs obtained from different degrees of PCE were also compared. Again the quadrature-based NIPC method described in the previous section was used to construct the response surface as a

TABLE 2: Sobol indices of each uncertain input in long-time averaged bubble size

\begin{tabular}{|ccc|}
\hline \hline Index & Parameter & Sobol indices \\
\hline \hline$S_{1}$ & $K$ & 0.9948 \\
$S_{2}$ & $U_{\infty}$ & 0.0022 \\
$S_{3}$ & $f$ & 0.0025 \\
\hline$S_{T_{1}}$ & $K$ & 0.9951 \\
$S_{T_{2}}$ & $U_{\infty}$ & 0.0026 \\
$S_{T_{3}}$ & $f$ & 0.0029 \\
\hline \hline
\end{tabular}


function of all three uncertain input variables. A degree convergence check study was performed where the PCE order was increased up to 4 and the response surface was constructed and evaluated at each order. Three locations (upstream of the separation bubble, inside the separation bubble, and downstream of the separation bubble), were selected to check the convergence of the expansion order of each output quantity in three different flow regions in the vicinity of the separation bubble. Figures 10-12 are the CDF plots showing the degree convergence of the PCE with respect to long-time averaged pressure and skin friction coefficients at the three selected locations $(x / c=0.62693$, upstream of the separation bubble; $x / c=0.994$, inside the separation bubble; and $x / c=1.5212$, downstream of the separation
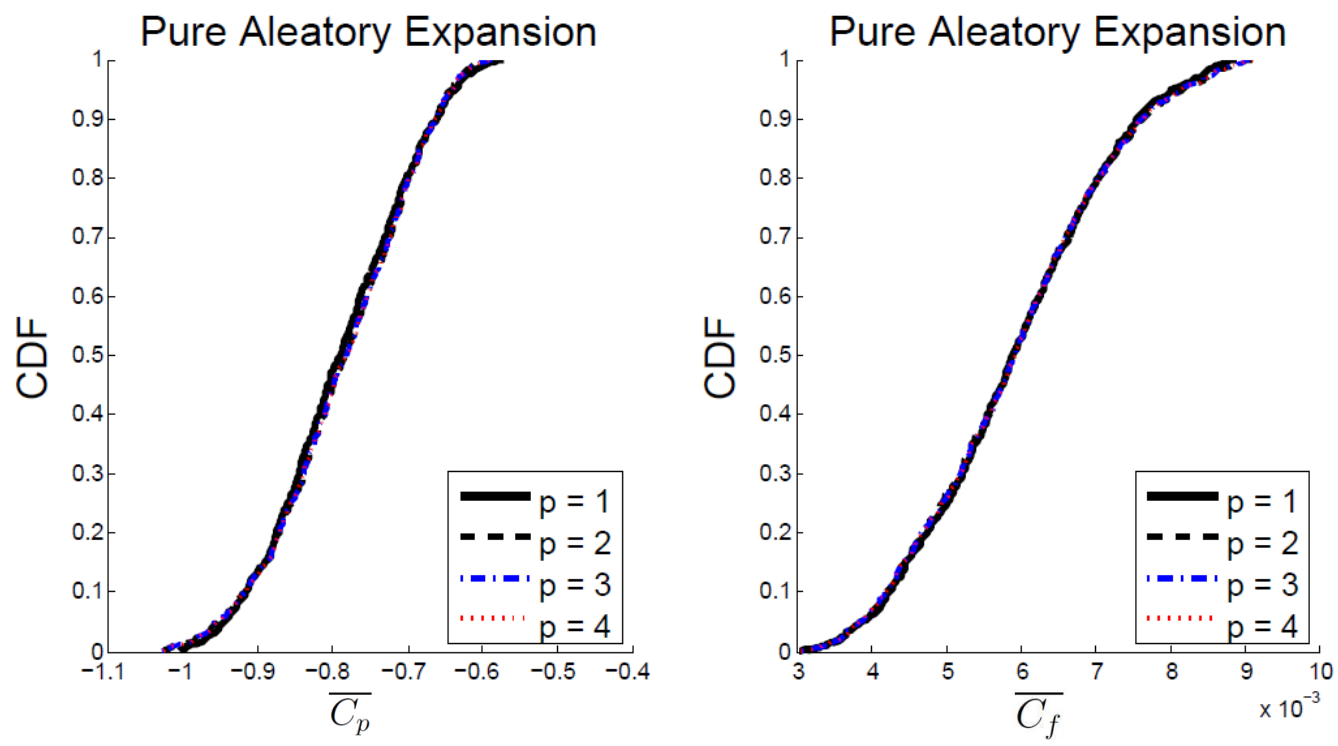

FIG. 10: PCE degree convergence check of long-time averaged pressure coefficient and skin-friction coefficient at location $x / c=0.62693$ (upstream of the separation bubble).
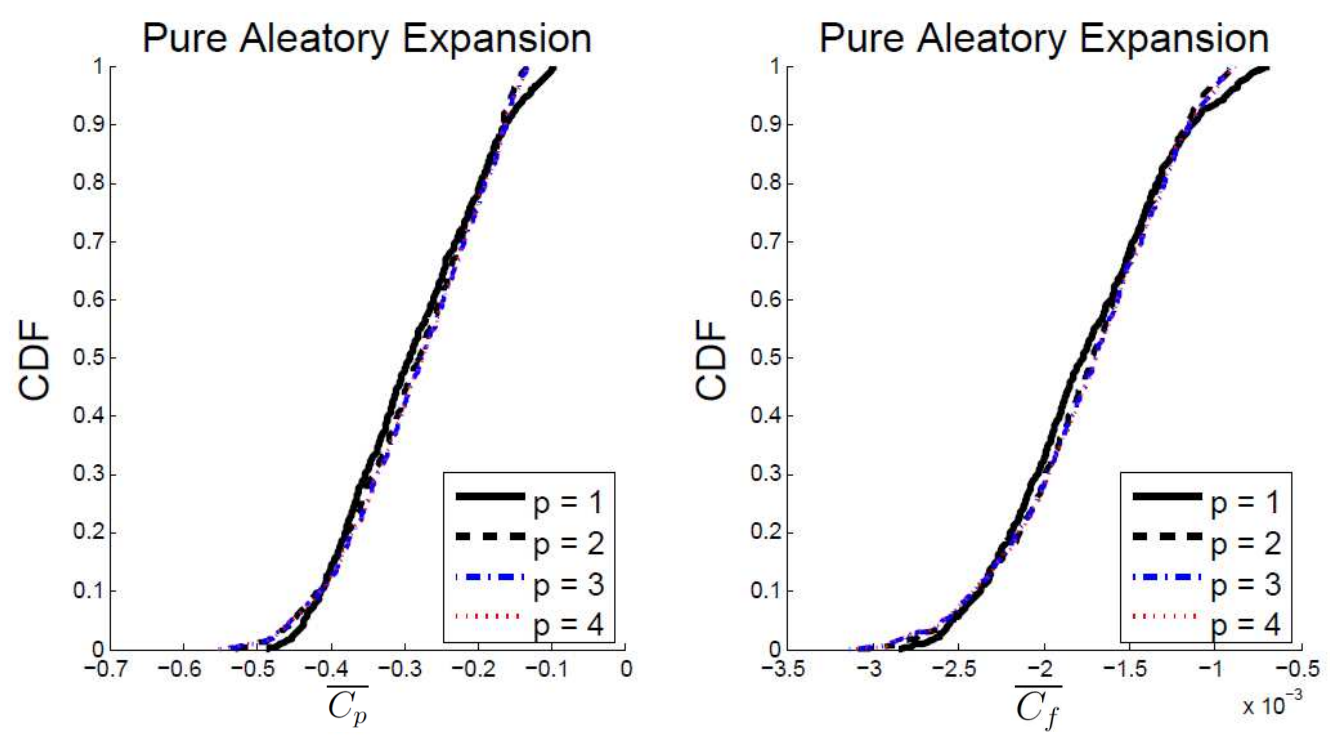

FIG. 11: PCE degree convergence check of long-time averaged pressure coefficient and skin-friction coefficient at location $x / c=0.994$ (inside the separation bubble). 

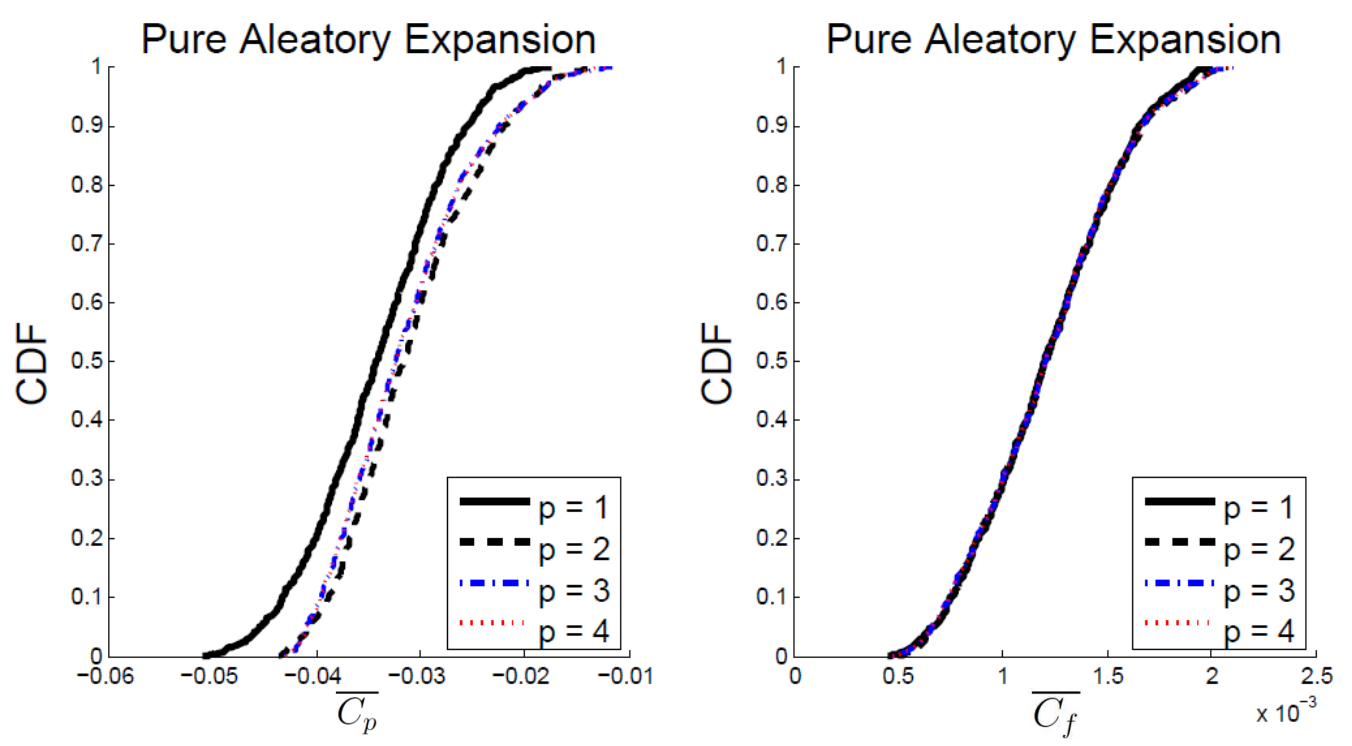

FIG. 12: PCE degree convergence check of long-time averaged pressure coefficient and skin-friction coefficient at location $x / c=1.5212$ (downstream of the separation bubble).

bubble), respectively. It is shown again that there is no obvious difference in the CDFs between third and fourth orders of PCE. Thus it can be concluded that the response surfaces obtained via the quadrature-based NIPC for long-time averaged pressure and skin friction coefficients at the selected locations again converge at the third order PCE.

\subsubsection{Results with Mixed (Aleatory-Epistemic) Uncertainty Assumption}

For the mixed aleatory-epistemic uncertainty quantification in long-time averaged pressure and skin friction coefficients along the wall, second-order probability approach was used again with the same response surface obtained for pure aleatory uncertainty assumption (fourth order PCE). One handred random samples from the specified bounds (Table 1) were selected for the epistemic uncertain variable in the outer loop. In the inner loop, for each specific value of the epistemic uncertain variable, 1000 randomly produced samples of aleatory uncertain variables based on the uniform probability distributions were utilized to evaluate the stochastic response surface. This procedure produced 100 CDFs which were then evaluated to find the lower and upper bounds of the interval at each probability level. The 95\% confidence intervals $(\mathrm{CI})$ of both pressure and skin friction coefficients were then calculated and plotted as shown in Fig. 13 (note that the axis for long-time averaged pressure coefficient is reversed). It is obvious that for long-time averaged pressure coefficient, the mixed uncertainty intervals are significant over the hump and near the separation bubble region. The $95 \% \mathrm{CI}$ for the long-time averaged skin-friction coefficients become significant starting from just downstream of the separation point indicating also the large uncertainty in the location of reattachment (i.e., where $\left.\overline{C_{f}}=0\right)$.

\subsubsection{Global Sensitivity Analysis with Sobol Indices}

The Sobol indices for the long-time averaged pressure and skin friction coefficients at the three selected locations were also computed with the fourth order PCE. These indices are shown in Tables 3-5, respectively. The results indicate again that the combined contributions of different uncertainty sources (e.g., $S_{1,2}$ ) are very small when comparing the total indices (e.g., $S_{T_{1}}$ ) with their corresponding individual (noncombined) indices (e.g., $S_{1}$ ) for both long-time averaged pressure and skin friction coefficients at all three locations. Furthermore, it can be seen that at a location upstream of the separation bubble (Table 3), the aleatory uncertainty input variable free stream velocity, $U_{\infty}$, is the 

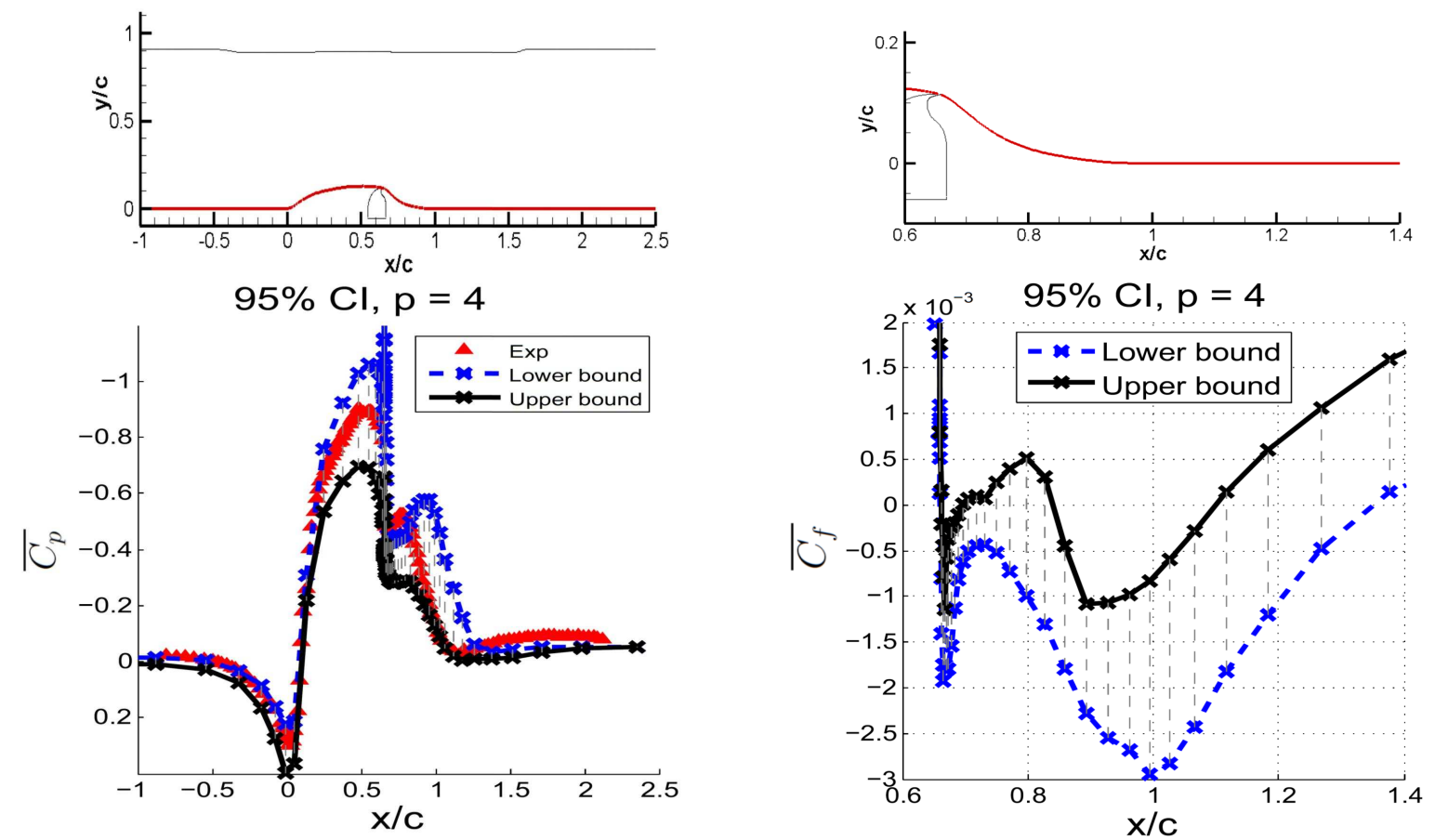

FIG. 13: $95 \%$ confidence interval for long-time averaged pressure and skin friction coefficients.

TABLE 3: Sobol indices of each uncertain input in long-time averaged pressure and skin friction coefficients at location $x / c=0.62693$ (upstream of the separation bubble)

\begin{tabular}{|cccc|}
\hline \hline Index & Parameter & Sobol indices for $\overline{C_{p}}$ & Sobol indices for $\overline{C_{f}}$ \\
\hline \hline$S_{1}$ & $K$ & 0.2535 & 0.7656 \\
$S_{2}$ & $U_{\infty}$ & 0.7423 & 0.2249 \\
$S_{3}$ & $f$ & $3.1460 \mathrm{E}-4$ & $6.1673 \mathrm{E}-5$ \\
\hline$S_{T_{1}}$ & $K$ & 0.2574 & 0.7750 \\
$S_{T_{2}}$ & $U_{\infty}$ & 0.7462 & 0.2343 \\
$S_{T_{3}}$ & $f$ & $3.3027 \mathrm{E}-4$ & $6.6998 \mathrm{E}-5$ \\
\hline \hline
\end{tabular}

TABLE 4: Sobol indices of each uncertain input in long-time averaged pressure and skin friction coefficients at location $x / c=0.994$ (inside the separation bubble)

\begin{tabular}{|cccc|}
\hline \hline Index & Parameter & Sobol indices for $\overline{C_{p}}$ & Sobol indices for $\overline{C_{f}}$ \\
\hline \hline$S_{1}$ & $K$ & 0.8805 & 0.6589 \\
$S_{2}$ & $U_{\infty}$ & 0.0951 & 0.2773 \\
$S_{3}$ & $f$ & $4.3054 \mathrm{E}-4$ & 0.0214 \\
\hline$S_{T_{1}}$ & $K$ & 0.9042 & 0.7002 \\
$S_{T_{2}}$ & $U_{\infty}$ & 0.1181 & 0.3134 \\
$S_{T_{3}}$ & $f$ & 0.0017 & 0.0291 \\
\hline \hline
\end{tabular}


TABLE 5: Sobol indices of each uncertain input in long-time averaged pressure and skin friction coefficients at location $x / c=1.5212$ (downstream of the separation bubble)

\begin{tabular}{|cccc|}
\hline \hline Index & Parameter & Sobol indices for $\overline{C_{p}}$ & Sobol indices for $\overline{C_{f}}$ \\
\hline \hline$S_{1}$ & $K$ & 0.5976 & 0.8272 \\
$S_{2}$ & $U_{\infty}$ & 0.3522 & 0.1611 \\
$S_{3}$ & $f$ & 0.0300 & 0.0024 \\
\hline$S_{T_{1}}$ & $K$ & 0.6102 & 0.8361 \\
$S_{T_{2}}$ & $U_{\infty}$ & 0.3721 & 0.1705 \\
$S_{T_{3}}$ & $f$ & 0.0385 & 0.0028 \\
\hline \hline
\end{tabular}

main contributor to the output uncertainty in long-time averaged pressure coefficient while at a location inside separation bubble (Table 4 ), the epistemic uncertainty variable, $K$ factor, becomes the main contributor. The contributions from $U_{\infty}$ and $K$ factor to the long-time averaged pressure coefficient are comparable at a location downstream of the separation bubble (Table 5). For long-time averaged skin-friction coefficient, the epistemic uncertainty input variable, $K$ factor, has a main contribution at all three selected locations. It is also noticeable that the uncertainty in the aleatory input variable frequency, $f$, has the least influence on both long-time averaged pressure and skin friction coefficients at all three selected locations.

\subsection{Uncertainty Quantification in Phase-Averaged Velocity Distributions}

For the phase-averaged quantities, the $\mathrm{x}$-velocity distributions at three selected locations were picked to perform the uncertainty quantification analysis. At locations of $x / c=0.66$ (just downstream of slot), $x / c=0.80$ (inside separation bubble) and $x / c=1.00$ (near the end of separation bubble), phase-averaged $\mathrm{x}$-velocity distributions at phase angles of $80^{\circ}, 170^{\circ}, 260^{\circ}$, and $350^{\circ}$ were analyzed with mixed (aleatory-epistemic) uncertainty assumption. Figure 14 shows a schematic of the three selected locations. The color in the contour represents the $\mathrm{x}$-velocity.

\subsubsection{Results with Mixed (Aleatory-Epistemic) Uncertainty Assumption}

Similar to the uncertainty quantification analysis approach for the long-time averaged pressure and skin-friction coefficients, phase-averaged $\mathrm{x}$-velocity distributions at selected locations were picked to perform the mixed uncertainty

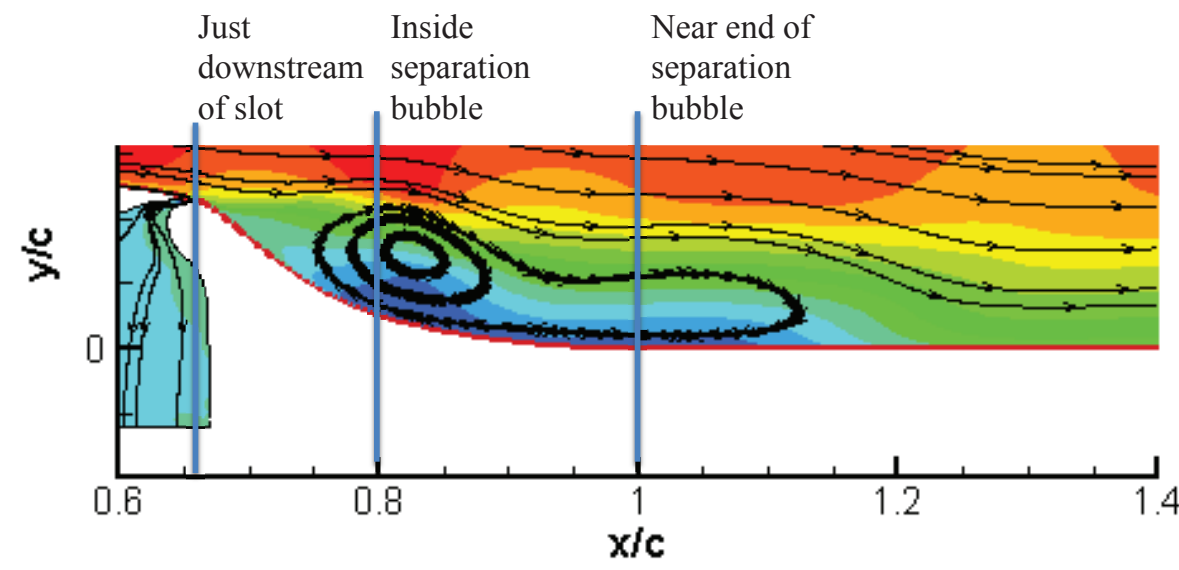

FIG. 14: Schematic of three selected locations to perform UQ analysis of phase-averaged $x$-velocity distributions. 
quantification. second-order probability approach was used again with a constructed stochastic response surface (fourth order PCE) to produce $100 \mathrm{CDFs}$ which was evaluated to find the lower and upper bounds of the interval at each probability level, as well as the $95 \%$ confidence intervals. Figures 15-17 show the second-order probability results for the phase-averaged $\mathrm{x}$-velocity distributions at locations of $x / c=0.66,0.80,1.00$, respectively. For comparison, the experimental data [2] are also included in the plots. The results show that with the uncertainty ranges considered, the output statistics are able to generally envelope the experimental data with $95 \%$ confidence intervals (CI) especially at locations of $x / c=0.66$ and $x / c=0.80$. At location of $x / c=1.00$, some of the experimental data close to the wall are still not captured by the confidence intervals at phase angles of $80^{\circ}$ and $350^{\circ}$. In addition to the uncertainty originating from the limitations of the eddy viscosity based turbulence models for separated flows (Ref. [2]), the uncertainty in the experimental measurements and other possible uncertainty sources not considered in the current study may be the reasons why the experimental data near the wall at this location are not captured within the confidence intervals obtained with the current uncertainty analysis.
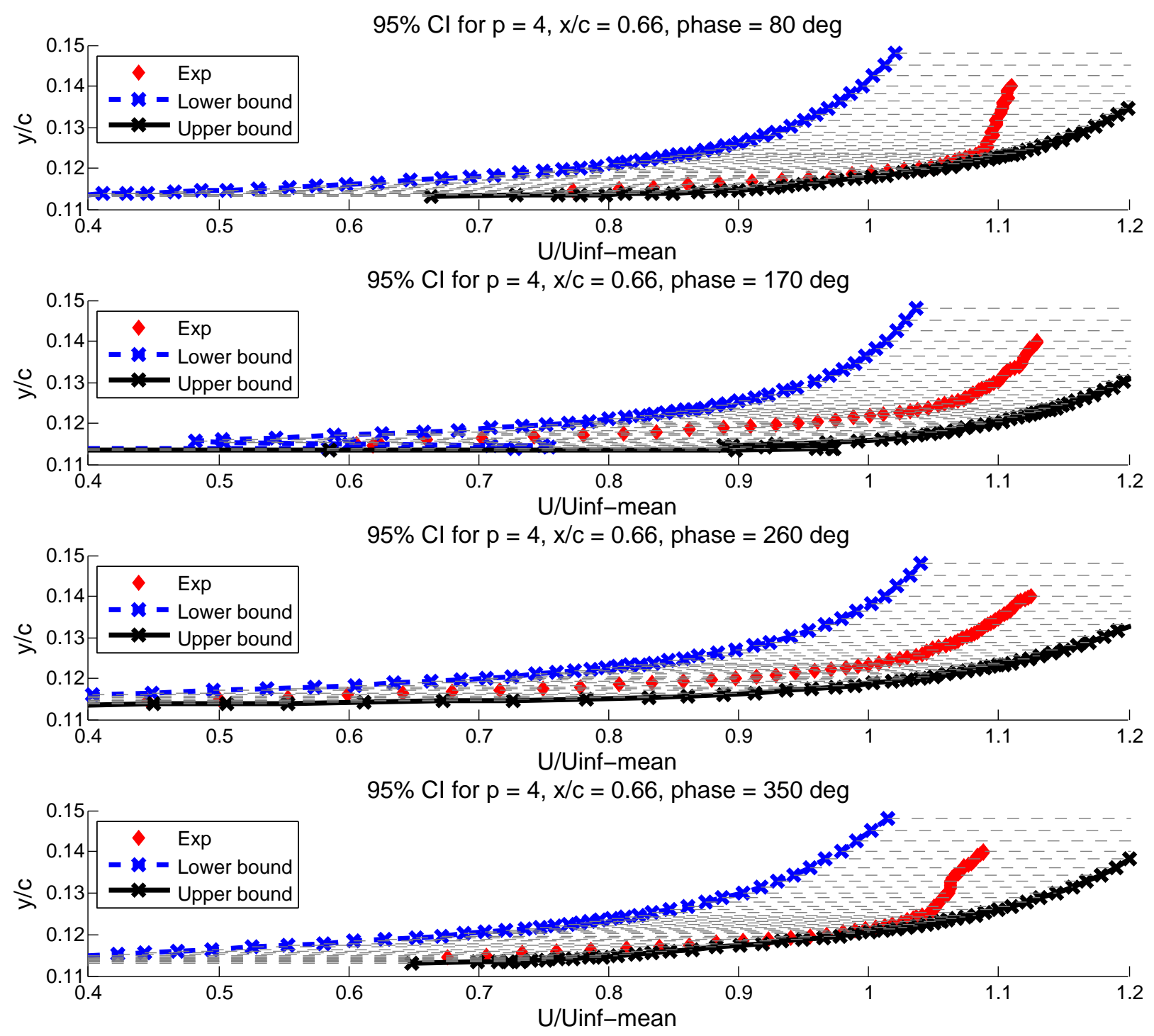

FIG. 15: $95 \% \mathrm{CI}$ for phase averaged $\mathrm{x}$-velocity distribution at location $x / c=0.66$. 

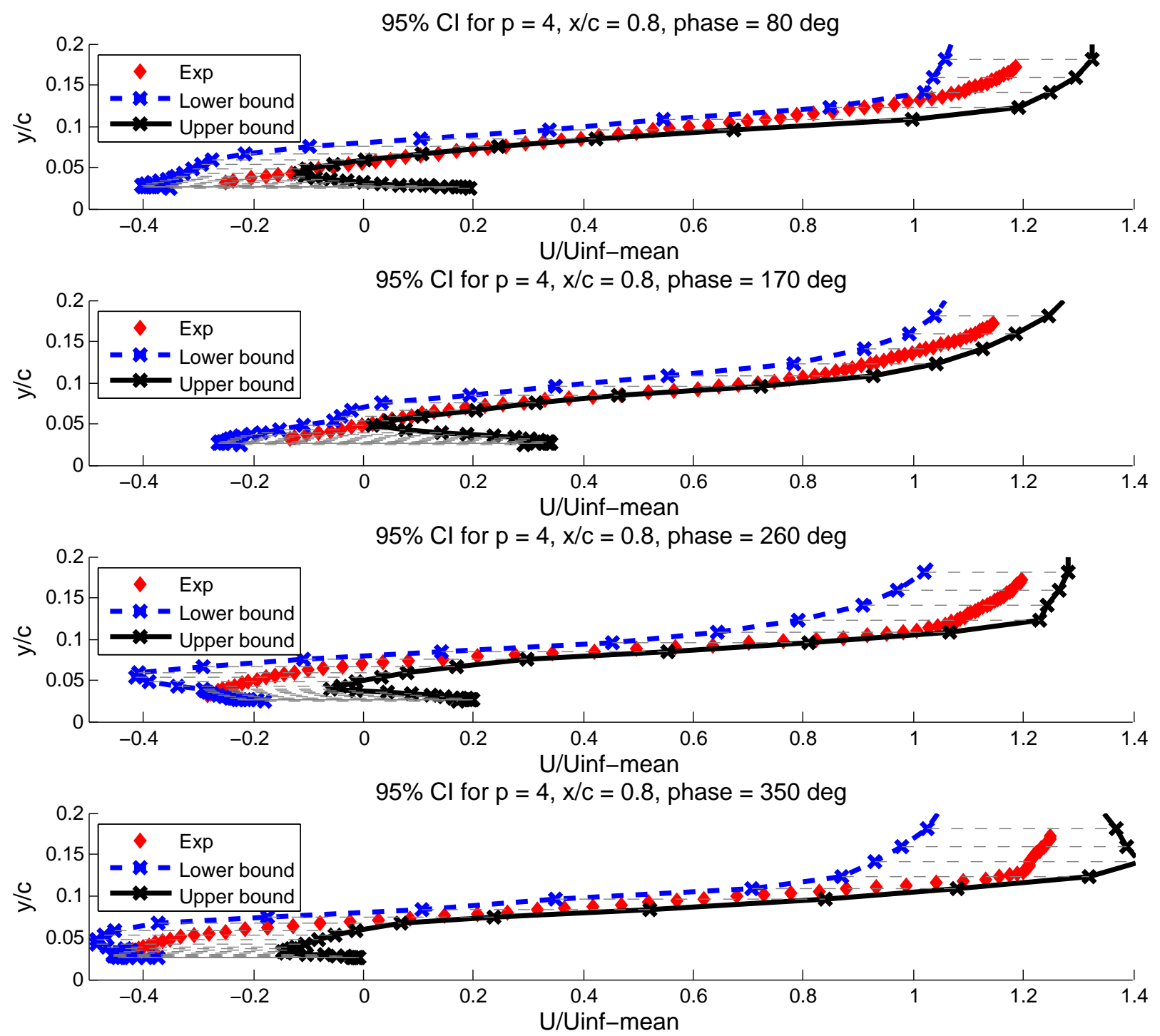

FIG. 16: $95 \% \mathrm{CI}$ for phase averaged $\mathrm{x}$-velocity distribution at location $x / c=0.80$.

\subsubsection{Global Sensitivity Analysis with Sobol Indices}

For the global sensitivity analysis, the $\mathrm{x}$-velocity distributions at three selected points at each location and phase angle were picked to calculate the Sobol indices with fourth order PCE. The results are tabulated in Tables 6-8, respectively.

The sensitivity analysis results show that, for each of the three $x / c$ locations, at a $y / c$ location near the wall, the epistemic uncertain input variable, $K$ factor (turbulence model), has the main contribution to the uncertainty in the $x$-velocity. Approaching the main stream, the contribution from the aleatory uncertain input variable, free stream velocity, $U_{\infty}$, becomes the dominant contributor to the uncertainty in the output x-velocity. The results also show that the contribution from the other aleatory uncertain variable, frequency, $f$, is significantly small compared to the other two uncertain variables for most regions of the velocity profile at three $x / c$ locations and four phase angles considered. 

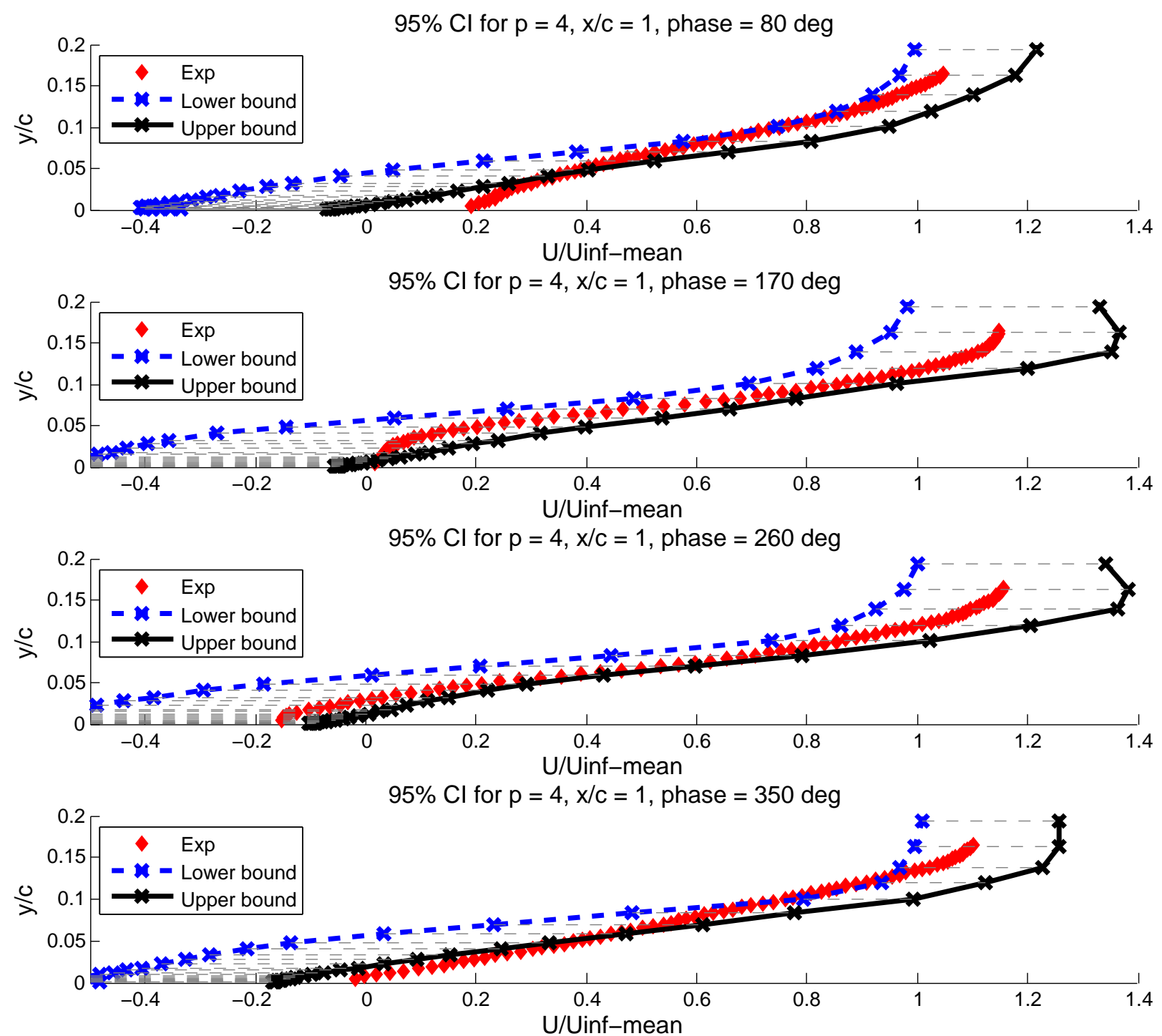

FIG. 17: $95 \% \mathrm{CI}$ for phase averaged $\mathrm{x}$-velocity distribution at location $x / c=1.00$.

\section{CONCLUSIONS}

In this study, an efficient methodology for the quantification of mixed (epistemic and aleatory) uncertainties in CFD modeling of synthetic jet actuators is introduced and demonstrated. The uncertainty quantification methodology is based on second-order probability theory implemented with a stochastic response surface obtained from quadraturebased nonintrusive polynomial chaos (NIPC). A global nonlinear sensitivity analysis is also utilized to quantify the contribution of each uncertainty source to the overall uncertainty in a selected output quantity. A test case, flow over a hump model with synthetic jet actuator control, was selected from the CFDVAL2004 workshop to demonstrate the application of the uncertainty quantification approach. Three uncertainty sources were considered: (1) epistemic (model-form) uncertainty in turbulence model by the introduction of a $K$ factor which is multiplied with the eddy viscosity coefficient in Spalart-Allmaras turbulence model, (2) aleatory (inherent) uncertainty in free stream velocity, and (3) aleatory uncertainty in actuation frequency. 
TABLE 6: Sobol indices for phase-averaged $\mathrm{x}$-velocity distribution at location $x / c=0.66$

\begin{tabular}{|c|c|c|c|c|c|}
\hline \multirow[b]{2}{*}{ Phase } & \multirow[b]{2}{*}{ Index } & \multirow[b]{2}{*}{ Parameter } & \multicolumn{3}{|c|}{$y / c$ Locations } \\
\hline & & & $\begin{array}{c}y / c=0.11322 \\
\text { (near } \\
\text { the wall) }\end{array}$ & $\begin{array}{c}y / c=0.11876 \\
\text { (between wall } \\
\text { and main stream) }\end{array}$ & $\begin{array}{c}y / c=0.14792 \\
\text { (near } \\
\text { main stream) }\end{array}$ \\
\hline \multirow{3}{*}{$80^{\circ}$} & $S_{T_{1}}$ & $K$ & 0.8663 & 0.2827 & 0.0026 \\
\hline & $S_{T_{2}}$ & $U_{\infty}$ & 0.1378 & 0.7167 & 0.9974 \\
\hline & $S_{T_{3}}$ & $f$ & 7.1667E-4 & 0.0013 & $1.1044 \mathrm{E}-4$ \\
\hline \multirow{3}{*}{$170^{\circ}$} & $S_{T_{1}}$ & K & 0.9961 & 0.5400 & 0.0032 \\
\hline & $S_{T_{2}}$ & $U_{\infty}$ & 0.0614 & 0.4631 & 0.9967 \\
\hline & $S_{T_{3}}$ & $f$ & 0.0031 & $4.8004 \mathrm{E}-4$ & $1.0968 \mathrm{E}-4$ \\
\hline \multirow{3}{*}{$260^{\circ}$} & $S_{T_{1}}$ & $K$ & 0.9919 & 0.7318 & 0.0012 \\
\hline & $S_{T_{2}}$ & $U_{\infty}$ & 0.0063 & 0.2654 & 0.9990 \\
\hline & $S_{T_{3}}$ & $f$ & 0.0272 & 0.0060 & $2.5496 \mathrm{E}-4$ \\
\hline \multirow{3}{*}{$350^{\circ}$} & $S_{T_{1}}$ & $K$ & 0.9123 & 0.4754 & 0.0091 \\
\hline & $S_{T_{2}}$ & $U_{\infty}$ & 0.0899 & 0.5249 & 0.9903 \\
\hline & $S_{T_{3}}$ & $f$ & 0.0012 & $6.0735 \mathrm{E}-4$ & $7.0411 \mathrm{E}-4$ \\
\hline
\end{tabular}

TABLE 7: Sobol indices for phase-averaged $\mathrm{x}$-velocity distribution at location $x / c=0.80$

\begin{tabular}{|c|c|c|c|c|c|}
\hline \multirow[b]{2}{*}{ Phase } & \multirow[b]{2}{*}{ Index } & \multirow[b]{2}{*}{ Parameter } & \multicolumn{3}{|c|}{$y / c$ Locations } \\
\hline & & & $\begin{array}{c}y / c=0.026463 \\
\text { (near } \\
\text { the wall) }\end{array}$ & $\begin{array}{c}y / c=0.10869 \\
\text { (between wall } \\
\text { and main stream) }\end{array}$ & $\begin{array}{c}y / c=0.20204 \\
\text { (near } \\
\text { main stream) }\end{array}$ \\
\hline \multirow{3}{*}{$80^{\circ}$} & $S_{T_{1}}$ & $K$ & 0.8786 & 0.8704 & 0.0258 \\
\hline & $S_{T_{2}}$ & $U_{\infty}$ & 0.1630 & 0.0230 & 0.9749 \\
\hline & $S_{T_{3}}$ & $f$ & 0.1969 & 0.1270 & 0.0029 \\
\hline \multirow{3}{*}{$170^{\circ}$} & $S_{T_{1}}$ & $K$ & 0.9122 & 0.4747 & 0.0025 \\
\hline & $S_{T_{2}}$ & $U_{\infty}$ & 0.0582 & 0.5184 & 0.9925 \\
\hline & $S_{T_{3}}$ & $f$ & 0.0719 & 0.0291 & 0.0060 \\
\hline \multirow{3}{*}{$260^{\circ}$} & $S_{T_{1}}$ & $\bar{K}$ & 0.9181 & 0.0932 & 0.0051 \\
\hline & $S_{T_{2}}$ & $U_{\infty}$ & 0.0712 & 0.8492 & 0.9957 \\
\hline & $S_{T_{3}}$ & $f$ & 0.0411 & 0.1492 & 0.0014 \\
\hline \multirow{3}{*}{$350^{\circ}$} & $S_{T_{1}}$ & $K$ & 0.5113 & 0.7053 & 0.0502 \\
\hline & $S_{T_{2}}$ & $U_{\infty}$ & 0.3834 & 0.4914 & 0.9449 \\
\hline & $S_{T_{3}}$ & $f$ & 0.1296 & 0.2004 & 0.0086 \\
\hline
\end{tabular}

The uncertainty quantification and sensitivity analysis results show that, for the uncertainty in long-time averaged separation bubble size, the $K$ factor which reflects the turbulence model uncertainty through the turbulent viscosity plays a dominant role compared to the other two uncertain input variables.

For the uncertainty in long-time averaged pressure coefficient, the free stream velocity, $U_{\infty}$, is the main contributor at a location upstream of the flow separation while at a location inside separation bubble, the epistemic uncertain variable $K$ becomes the main contributor. The contributions from $U_{\infty}$ and $K$ factor are comparable at a location 
TABLE 8: Sobol indices for phase-averaged $\mathrm{x}$-velocity distribution at location $x / c=1.00$

\begin{tabular}{|cccccc|}
\hline \hline Phase & Index & Parameter & $\begin{array}{c}y / c=0.0064552 \\
\text { (near } \\
\text { the wall) }\end{array}$ & $\begin{array}{c}y / c=0.082942 \\
\text { (between wall } \\
\text { and main stream) }\end{array}$ & $\begin{array}{c}y / c=0.19335 \\
\text { (near } \\
\text { main stream) }\end{array}$ \\
\hline \hline \multirow{3}{*}{$80^{\circ}$} & $S_{T_{1}}$ & $K$ & 0.8262 & 0.3108 & 0.0209 \\
& $S_{T_{2}}$ & $U_{\infty}$ & 0.0595 & 0.7156 & 0.9679 \\
& $S_{T_{3}}$ & $f$ & 0.1416 & 0.0306 & 0.0204 \\
\hline \hline \multirow{3}{*}{$170^{\circ}$} & $S_{T_{1}}$ & $K$ & 0.8711 & 0.7879 & 0.1172 \\
& $S_{T_{2}}$ & $U_{\infty}$ & 0.1401 & 0.1791 & 0.8708 \\
& $S_{T_{3}}$ & $f$ & 0.0767 & 0.0945 & 0.0320 \\
\hline \hline \multirow{3}{*}{$260^{\circ}$} & $S_{T_{1}}$ & $K$ & 0.7492 & 0.5848 & 0.1554 \\
& $S_{T_{2}}$ & $U_{\infty}$ & 0.1823 & 0.4050 & 0.8411 \\
& $S_{T_{3}}$ & $f$ & 0.0797 & 0.0230 & 0.0106 \\
\hline \hline \multirow{3}{*}{$350^{\circ}$} & $S_{T_{1}}$ & $K$ & 0.9296 & 0.3370 & 0.0708 \\
& $S_{T_{2}}$ & $U_{\infty}$ & 0.0426 & 0.6718 & 0.9077 \\
& $S_{T_{3}}$ & $f$ & 0.0656 & 0.0378 & 0.0366 \\
\hline \hline
\end{tabular}

downstream of the flow separation. For the uncertainty in long-time averaged skin-friction coefficient, the $K$ factor has the main contribution at all three selected locations.

The sensitivity analysis results show that, for each of the three $x / c$ locations, at a $y / c$ location near the wall, the epistemic uncertain input variable, $K$ factor (turbulence model), has the main contribution to the uncertainty in the $\mathrm{x}$-velocity. Approaching the main stream, the contribution from the free stream velocity becomes the dominant contributor to the uncertainty in the $\mathrm{x}$-velocity. The results also show that the contribution from the other aleatory uncertain variable, frequency, $f$, is significantly small compared to the other two uncertain variables for most regions of the velocity profile at three $x / c$ locations and four phase angles considered.

Although we focus on the uncertainty quantification of a synthetic jet problem in the current study, the mixed uncertainty quantification approach demonstrated in this study can also be applied to other computational fluid dynamics problems with inherent and model-form input uncertainties.

\section{REFERENCES}

1. Glezer, A. and Amitay, M., Synthetic jets, Ann. Rev. Fluid Mech., 34:503-529, 2002.

2. Nasa Langley Research Center Workshop: CFD validation of synthetic jets and turbulent separation control, http://cfdval2004.larc.nasa.gov/, March 2004.

3. Rumsey, C. L., Gatski, T. B., Sellers, W. L., III, Vatsa, V. N., and Viken, S. A., Summary of the 2004 computational fluid dynamics validation workshop on synthetic jets, AIAA J., 44(2):194-207, 2006.

4. Rumsey, C. L., Successes and challenges for flow control simulations (invited), AIAA 2008-4311, In 4th AIAA Flow Control Conference, Seattle, WA, June 2008.

5. Bettis, B. R. and Hosder, S., Efficient uncertainty quantification approach for reentry flows with mixed uncertainties, J. Thermophys. Heat Transfer, 25(4):523-535, 2011.

6. Hosder, S. and Bettis, B. R., Uncertainty and sensitivity analysis for reentry flows with inherent and model-form uncertainties, J. Spacecr. Rockets, 49(2):193-206, 2012.

7. West, T. K., Hosder, S., and Johnston, C. O., Multistep uncertainty quantification approach applied to hypersonic reentry flows, J. Spacecr. Rockets, 51(1):296-310, 2014. 
8. Hosder, S. and Walters, R. W., Non-Intrusive polynomial chaos methods for uncertainty quantification in fluid dynamics, AIAA 2010-129, In 48th AIAA Aerospace Sciences Meeting Including the New Horizons Forum and Aerospace Exposition, Orlando, FL, January 2010.

9. Adya, S., Han, D., and Hosder, S., Uncertainty quantification integrated to cfd modeling of synthetic jet actuators, Int. J. Flow Control, 2(3):169-181, 2010 .

10. Oberkampf, W. L., Helton, J. C., and Sentz, K., Mathematical representation of uncertainty, AIAA 2001-1645, In 3rd NonDeterministic Approaches Forum, Seattle, WA, April 2001.

11. Oberkampf, W. L. and Helton, J. C., Investigation of evidence theory for engineering applications, AIAA 2002-1569, In 4th Non-Deterministic Approaches Forum, Denver, CO, April 2002.

12. Eldred, M. and Swiler, L., Efficient algorithms for mixed Aleatory-Epistemic uncertainty quantification with application to Radiation-Hardened electronics, Sandia National Laboratories Report, SAND2009-5805, September 2009.

13. Swiler, L., Paez, T., Mayes, R., and Eldred, M., Epistemic uncertainty in the calculation of margins, AIAA 2009-2249, In 50th AIAA/ASME/ASCE/AHS/ASC Structures, Structural Dynamics, and Materials Conference, Palm Springs, CA, May 2009.

14. Bettis, B. and Hosder, S., Quantification of uncertainty in aerodynamic heating of a reentry vehicle due to uncertain wall and freestream conditions, AIAA 2011-252, In 49th AIAA Aerospace Sciences Meeting Including the New Horizons Forum and Aerospace Exposition, Orlando, FL, January 2011.

15. Wiener, N., The homogeneous chaos, Am. J. Math., 60(4):897-936, 1938.

16. Xiu, D. and Karniadakis, G. E., Modeling uncertainty in flow simulations via generalized polynomial chaos, J. Comput. Phys., 187(1):137-167, 2003.

17. Eldred, M. S., Webster, C. G., and Constantine, P. G., Evaluation of Non-Intrusive approaches for Wiener-Askey generalized polynomial chaos, AIAA 2008-1892, In 10th AIAA Non-Deterministic Approaches Forum, Schaumburg, IL, April 2008.

18. Walters, R. W. and Huyse, L., Uncertainty analysis for fluid mechanics with applications, Tech. Rep., ICASE 2002-1, NASA/CR-2002-211449, NASA Langley Research Center, Hampton, VA, 2002.

19. Najm, H. N., Uncertainty quantification and polynomial chaos techniques in computational fluid dynamics, Ann. Rev. Fluid Mech., 41:35-52, 2009.

20. Sobol, I., Global sensitivity indices for nonlinear mathematical models and their Monte Carlo estimates, Math. Comput. Simul., 55:271-280, 2001

21. Sudret, B., Global sensitivity analysis using polynomial chaos expansion, Reliabil. Eng. Syst. Safety, 93(7):964-979, 2008.

22. Crestaux, T., Maitre, O. L., and Martinez, J.-M., Polynomial chaos expansion for sensitivity analysis, Reliabil. Eng. Syst. Safety, 94(7):1161-1172, 2009.

23. Ghaffari, S., Magin, T., and Iaccarino, G., Uncertainty quantification of radiative heat flux modeling for titan atmospheric entry, AIAA 2010-239, In 48th AIAA Aerospace Sciences Meeting Including the New Horizons Forum and Aerospace Exposition, Orlando, FL, January 2010.

24. ANSYS FLUENT Manual.

25. Spalart, P. R. and Allmaras, S. R., A one-equation turbluence model for aerodynamic flows, La Recherche Aerospatiale, (1):5-21, 1994.

26. Rumsey, C. L., Reynolds-averaged Navier-Stokes analysis of zero efflux flow control over a hump model, AIAA 2006-1114, In 44th AIAA Aerospace Sciences Meeting and Exhibit, Reno, NV, January 2006. 